ARTICLE

\title{
Rationally designed bacterial consortia to treat chronic immune-mediated colitis and restore intestinal homeostasis
}

Daniel van der Lelie (1) ${ }^{1 凶}$, Akihiko Oka (D) ${ }^{2,3}$, Safiyh Taghavi ${ }^{1}$, Junji Umeno ${ }^{2,4}$, Ting-Jia Fan', Katherine E. Merrell (1) ${ }^{1}$, Sarah D. Watson', Lisa Ouellette (1) ${ }^{1}$, Bo Liu ${ }^{2}$, Muyiwa Awoniyi ${ }^{2}$, Yunjia Lai (1) ${ }^{5}$, Liang $\mathrm{Chi}^{5}$, Kun Lu ${ }^{5}$, Christopher S. Henry ${ }^{1} \&$ R. Balfour Sartor ${ }^{2}$

Environmental factors, mucosal permeability and defective immunoregulation drive overactive immunity to a subset of resident intestinal bacteria that mediate multiple inflammatory conditions. GUT-103 and GUT-108, live biotherapeutic products rationally designed to complement missing or underrepresented functions in the dysbiotic microbiome of IBD patients, address upstream targets, rather than targeting a single cytokine to block downstream inflammation responses. GUT-103, composed of 17 strains that synergistically provide protective and sustained engraftment in the IBD inflammatory environment, prevented and treated chronic immune-mediated colitis. Therapeutic application of GUT-108 reversed established colitis in a humanized chronic $\mathrm{T}$ cell-mediated mouse model. It decreased pathobionts while expanding resident protective bacteria; produced metabolites promoting mucosal healing and immunoregulatory responses; decreased inflammatory cytokines and Th-1 and Th-17 cells; and induced interleukin-10-producing colonic regulatory cells, and IL-10independent homeostatic pathways. We propose GUT-108 for treating and preventing relapse for IBD and other inflammatory conditions characterized by unbalanced microbiota and mucosal permeability.

\footnotetext{
${ }^{1}$ Gusto Global LLC, Morrisville, NC, USA. ${ }^{2}$ Departments of Medicine, Microbiology and Immunology, Center for Gastrointestinal Biology and Disease, University of North Carolina at Chapel Hill, Chapel Hill, NC, USA. ${ }^{3}$ Department of Internal Medicine II, Shimane University Faculty of Medicine, Shimane, Japan. ${ }^{4}$ Department of Medicine and Clinical Science, Graduate School of Medical Sciences, Kyushu University, Fukuoka, Fukuoka, Japan. ${ }^{5}$ Department of Environmental Sciences and Engineering, Gillings School of Public Health, University of North Carolina at Chapel Hill, Chapel Hill, NC, USA.

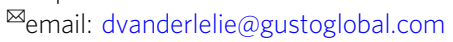


C hronic intestinal inflammation can be induced by multiple exogenous and endogenous signals and is mediated by immune and nonimmune cells in genetically susceptible hosts with defects in epithelial barrier function, immunoregulation, or bacterial killing. Exogenous substances including dietary products, pathogenic microorganisms, xenobiotics including antibiotics, or various combinations thereof, can trigger initial mucosal injury and/or dysbiosis to initiate acute intestinal inflammation that is perpetuated by the antigenic activities of a subset of resident microbiota $^{1,2}$. Examples of these conditions are inflammatory bowel diseases (IBD), which encompass two main clinical disorders: Crohn's disease and ulcerative colitis. Current IBD treatments primarily control inflammation through anti-inflammatory and immunosuppressive mechanisms. Some of the most successful drugs for treating IBD include infliximab, adalimumab, vedolizumab, ustekinumab, and tofacitinib, which target specific immune components to control the inflammatory process. However, these and other immune modulating drugs induce sustained, steroid-free remission in only a small subset of patients and can have multiple serious side effects, including an increased risk for serious and potentially life-threatening infections and neoplasia. In addition, these drugs do not correct upstream conditions that contribute to the chronic inflammatory mechanisms, including the leaky mucosal barrier, a pro-inflammatory gut microbiome and immunoregulatory defects.

As an alternative to anti-inflammatory and immunosuppressive therapies, microbiome-inspired live biotherapeutic products (LBPs) are being developed to treat conditions linked to chronic intestinal inflammation and increased permeability. The traditional approach for LBP discovery has been to compare the microbiomes of healthy subjects and patients suffering from a specific condition, such as IBD, to identify microorganisms that are lacking or under-represented in large databases, such as the HMP2 project $^{3}$. This information, further enforced by the results from microbial association studies, is used to propose a therapeutic formulation to replenish the microorganisms that are lacking or under-represented ${ }^{2,4}$. In the case of IBD, early efforts have focused on the use of strains belonging to the Clostridium clusters IV and XIVa, which were found to successfully decrease inflammation in rodent IBD models ${ }^{5-7}$. Using germ-free (GF) mice inoculated with healthy human fecal material pretreated with chloroform to enrich for spore-forming bacteria, a stable 17strain consortium was enriched from a single donor based on their ability to induce colonic regulatory $\mathrm{T}$ cells (Tregs) 7,8 . This consortium was comprised of spore-forming Clostridium cluster IV, XIVa, and XVIII strains that produced butyrate and decreased the severity of several colitis models ${ }^{7}$.

Open label application of Fecal Microbiome Transplants and enrichment-based approaches have several disadvantages. The outcome is defined by the stool sample used for the enrichment, with different samples representing different consortia with variable efficacy; ${ }^{9}$ undesirable strains/functions associated with safety risks including virulence factors and transferable antibiotic resistance functions might be present, such as the presence of enteropathogenic and Shigatoxin-producing Escherichia coli strains ${ }^{10}$ and antibiotic-resistant E. coli strains ${ }^{11}$ in FMTs, or the presence of transferable vancomycin resistance elements as found in the genome of the VE202 consortium strain Blautia coccoides VE202-06 (GenBank Accession Number Accession: PRJDB525). Furthermore, consortium modeling, as presented in this study, shows that other bacterial species besides spore-forming Clostridium bacteria provide metabolic support and additional therapeutic functions required for optimal engraftment and therapeutic performance of live biotherapeutic products in the hostile gut environment of patients with intestinal inflammation. These shortcomings can be addressed by a bottom-up rational consortium design approach that is rigorously informed by mechanistic modeling and insights from microbiome ecology and disease pathogenesis. We used this approach to combine wellcharacterized strains isolated from many healthy human stool samples into a consortium of metabolically interdependent strains with a variety of therapeutic functionalities being distributed in a redundant way between strains. Initially a 17-strain consortium, GUT-103, was designed around publicly available strains. GUT-103 rapidly colonized mice, restored normal function to the inflamed colon, and prevented and reversed established experimental colitis in gnotobiotic mice. Based on these proof of concept studies, a refined 11-member consortium, GUT108 , was designed around a panel of proprietary human bacterial strains that strongly engrafted and provided similar redundant protective functions. Therapeutically applied GUT-108 corrected functional dysbiosis of the inflamed gut microbiome and treated established colitis in a humanized mouse colitis model while decreasing opportunistic pathogenic bacteria, increasing resident protective bacterial groups, and restoring immunologic and metabolic homeostasis.

\section{Results}

Rational design of the GUT-103 consortium. Using a bottomup approach, LBPs were rationally designed to provide key therapeutic functions involved in mucosal homeostasis and immune modulation defective in the gut microbiome of IBD patients ${ }^{2,3}$. These functions include synthesis of short-chain fatty acids (SCFA) including butyrate and propionate, synthesis of indole, indole- 3 propionic acid (IPA) and indole- 3 acetic acid (IAA) from tryptophan, and deconjugation of bile salts and their conversion into therapeutic secondary bile acids, especially deoxycholic acid (DCA) and lithocholic acid (LCA). Additional functions included to control pathobionts were the synthesis of antimicrobials and siderophores and the uptake of heterologous siderophores produced by Enterobacteriaceae including Escherichia, Klebsiella, and Shigella species, allowing bacteria to compete with these opportunistic pathogens for the essential nutrient iron. The importance of the gut microbiome to provide these specific functions ${ }^{12-14}$ and the role of specific microbes encoding key activities, such as the $7 \alpha / \beta$-dehydroxylation bile acid conversion pathway encoded by Clostridium scindens ${ }^{15}$ has been extensively reviewed.

Next, commensal human gut bacteria that can provide these desired functions were identified. Reference isolate genome sequences for common human gut microbes were collected based on published gut $16 \mathrm{~S}$ rRNA studies and isolate source metadata (https://www.vmh.life/\#home). Isolate and metagenome assembled genome (MAG) sequences were gathered from RefSeq ${ }^{16}$, PATRIC $^{17}$ and the Human Microbiome Project ${ }^{18}$. Consistently annotated genomes were used to determine which genomes were likely to encode proteins with the desired therapeutic functions. Comparative analysis of annotations from these genomes also enabled the identification of genera where selected therapeutic functions were highly conserved. A key finding from this analysis was that members of the Clostridium clusters IV and XIVa families could not provide several critical functionalities required to restore the proinflammatory microbiome of IBD patients, including the synthesis of propionate and indole-based derivatives from tryptophan. Therefore, the rational design of LBPs to restore these critical functionalities should also include members of the families Bacteroidaceae, Eubacteriaceae, Akkermansiaceae, and non-Clostridium clusters IV and XIVa family members. Ultimately, a set of the most desirable genera were selected from which to compose the rationally designed LBPs, including isolates belonging to the genera Akkermansia (Supplementary Data 1), Bacteroides (Supplementary 
Data 2), Clostridium (Supplementary Data 3), and Faecalibacterium (Supplementary Data 4). Multi-genome analysis for the genera Akkermansia and Faecalibacterium combined with whole-genome DNA average nucleotide identity (ANI) showed that species previously classified as Akkermansia muciniphila and Faecalibacterium prausnitzii are comprised of several species, distinguished by over $10 \%$ difference in whole-genome DNA sequence compared to their type strains.

Although this comparative analysis identified desirable genera, it was still necessary to identify specific strains within each genus to comprise the consortia. In this selection process, it is most important to identify strains with complementary auxotrophies for essential amino acids, vitamins, and co-factors. This was based on previous analyses of natural microbiomes, which revealed that co-evolved strains in these systems often have complementary auxotrophies ${ }^{19}$, and addresses the risk of one strain outcompeting the other members of the consortium. Furthermore, redundancy in metabolic dependencies between strains is important to avoid that loss of one strain will not lead to the collapse of the consortium. Auxotrophic analysis of isolate strain genomes and MAGs from our selected genera revealed that auxotrophies were typically conserved among most members of the same species, but with enough variation to make this analysis important to include in the design process.

Overall, using redundancy in key functionalities combined with a distribution of complementary auxotrophies to create a network of metabolically interdependent strains, a consortium of 17 publicly available human strains was selected, referred to as GUT-103 (Table 1). For each of the strains, individual strain models were constructed that were subsequently combined into a consortium model. Flux Balance analysis was used to predict in silico that the GUT-103 strains form a network of metabolic dependencies complementing each other's metabolic needs, indicating that GUT-103 can function as a consortium without the need for auxotrophies being complemented by resident bacteria from the imbalanced gut microbiome of IBD patients ${ }^{3}$. The GUT-103 functional characteristics, auxotrophy reactions, flux balance analysis, and strain interaction table to predict the growth of the individual GUT-103 strains and the GUT-103 consortium under defined conditions are shown in Supplementary Data 5.

GUT-103 successfully prevents and treats experimental colitis. Preliminary testing using quantitative PCR with species-specific $r p o B$ gene primers showed that 2 weeks after gavage GUT-103 successfully colonized GF 129 WT and 129 interleukin-10 deficient $\left(I l 10^{-/-}\right)$mice (see Supplementary Fig. 1a, b and Supplementary Table 1). Three human bacteria, Escherichia coli LF82, Enterococcus faecalis OG1RF, and Ruminococcus gnavus ATCC29149 (EER strains) were used based on their ability to colonize GF $129 \mathrm{WT}$ and $129 \mathrm{Il10^{-/ }}$ mice (Supplementary Fig. 2a, b and Supplementary Table 2), to induce chronic colitis and to activate strain-specific Th1 and Th17 mucosal immune responses in gnotobiotic $I l 10^{-/-}$mice ${ }^{20,21}$. Selective colonization of gnotobiotic $1 l 10^{-/}$mice by EER showed increased fecal lipocalin-2 concentrations $(149.8 \pm 80.2 \mathrm{ng} / \mathrm{g}$ feces, a $2 \mathrm{log}$ increase from baseline values) at 2 weeks, with further progression over 4 weeks (Supplementary Fig. 3). Based on these kinetic studies showing mildly active colitis 2 weeks after EER colonization, we chose 2 weeks for GUT-103 therapeutic intervention. The ability of GUT-103 to colonize, compete with IBD-relevant pathobionts and to both prevent and treat establish Th1/Th17mediated colitis was subsequently tested (Fig. 1a). GUT-103 was successfully established one week after gavage in EER $1110^{-/-}$ mice, (Fig. 1b) with a composition similar to that in ex-GF mice
(Supplementary Fig. 1). All strains were detected but concentrations varied. Three dominant strains, Blautia producta DSM2950, Akkermansia muciniphila ATCC BAA-835 and Bacteroides massiliensis DSM17679, with complementary auxotrophies, suggest the formation of an integrated metabolic network. Relatively high levels of Bacteroides stercoris DSM19555, which has similar auxotrophies and functions as Bacteroides massiliensis DSM17679, were also observed. These predominant community strains are predicted to synthesize SCFA and indole, but lack synthesis of siderophores, bacteriocin synthesis and bile acid conversion. GUT-103 decreased concentrations of each of the EER strains in aggregate to less than $20 \%$ of the total bacterial population, even in the therapeutic protocol where the three colitogenic strains were established for 2 weeks (Fig. 1b). The preventive protocol blocked R. gnavus concentrations to below $1 \%$.

Colonization with GUT-103 did not induce inflammation, but both the preventive and therapeutic protocols significantly decreased colitis in EER-inoculated $I l 10^{-1-}$ mice compared with PBS controls, as measured by lipocalin-2 levels (Fig. 1c), crypt hyperplasia and lamina propria (LP) mononuclear cell infiltration (Fig. 1d), interaction table predict the growth of the individual GUT-108 and blinded histology scoring (Fig. 1e). Gavage with GUT-103 also increased synthesis of butyrate and propionate that was deficient in EER-inoculated $I l 10^{-/-}$mice (Fig. 1f) and decreased inflammation measured by cecal mRNA expression for IFN $\gamma$, IL-12p40, and TNFa (Fig. 1g).

Bile acid profiles were analyzed to further evaluate restoration of a functional gut microbiome by engraftment with GUT-103 strains. Significantly higher levels of multiple unconjugated bile acids were observed in cecal contents from Therapy, Prevention and GUT-103 groups compared with EER-inoculated $1 l 10^{-/-}$ mice (Fig. 2a). Compared to EER, therapeutic application of GUT-103 increased cecal luminal concentrations of the unconjugated primary bile acids $\beta \mathrm{MCA}, \mathrm{CA}$, and CDCA. Cecal contents of the GUT-103 group had higher levels of the secondary bile acids UDCA and LCA than EER-inoculated $I l 10^{-/-}$mice, but low DCA levels were not significantly different between these groups (Fig. 2a). In contrast to free bile acids, the cecal luminal levels of multiple conjugated bile acids, including T $\beta$ MCA, TCDCA, TUDCA, and GCA, in all GUT-103 groups were significantly lower than those in EER (Fig. 2b). However, the level of glycineconjugated GLCA was significantly higher in the GUT-103colonized group than in the EER group (Fig. 2b). Overall, compared to EER, GUT-103 decreased levels of conjugated bile acids especially for taurine-conjugated bile acids. Together, these results demonstrate both protective and therapeutic activities of GUT-103 and in vivo validation of the anticipated replacement of pathobionts and protective metabolic functions of this rationally designed consortium. It should also be noted that in preliminary studies we demonstrated that two independent consortia of 14 and 19 bacterial isolates, respectively, from two healthy human volunteers induced colitis when colonized into germ-free $129 \mathrm{IllO}^{-/-}$mice $^{22,23}$.

Design of the optimized GUT-108 consortium. Informed by the GUT-103 outcomes, we rationally designed an optimized 11-strain consortium referred to as GUT-108 (Table 2). Compared to GUT-103, GUT-108 included improved redundancy for synthesis of the therapeutic secondary bile acids LCA and DCA covered by Extibacter sp. GGCC_0201; yersiniabactin synthesis provided by Clostridium symbiosum GGCC_0272; and several strains with functions antagonistic against opportunistic pathogens into the Enterobacteriaceae family. The GUT-108 functional characteristics, auxotrophy reactions, flux balance analysis, and strain interaction table predict the growth 


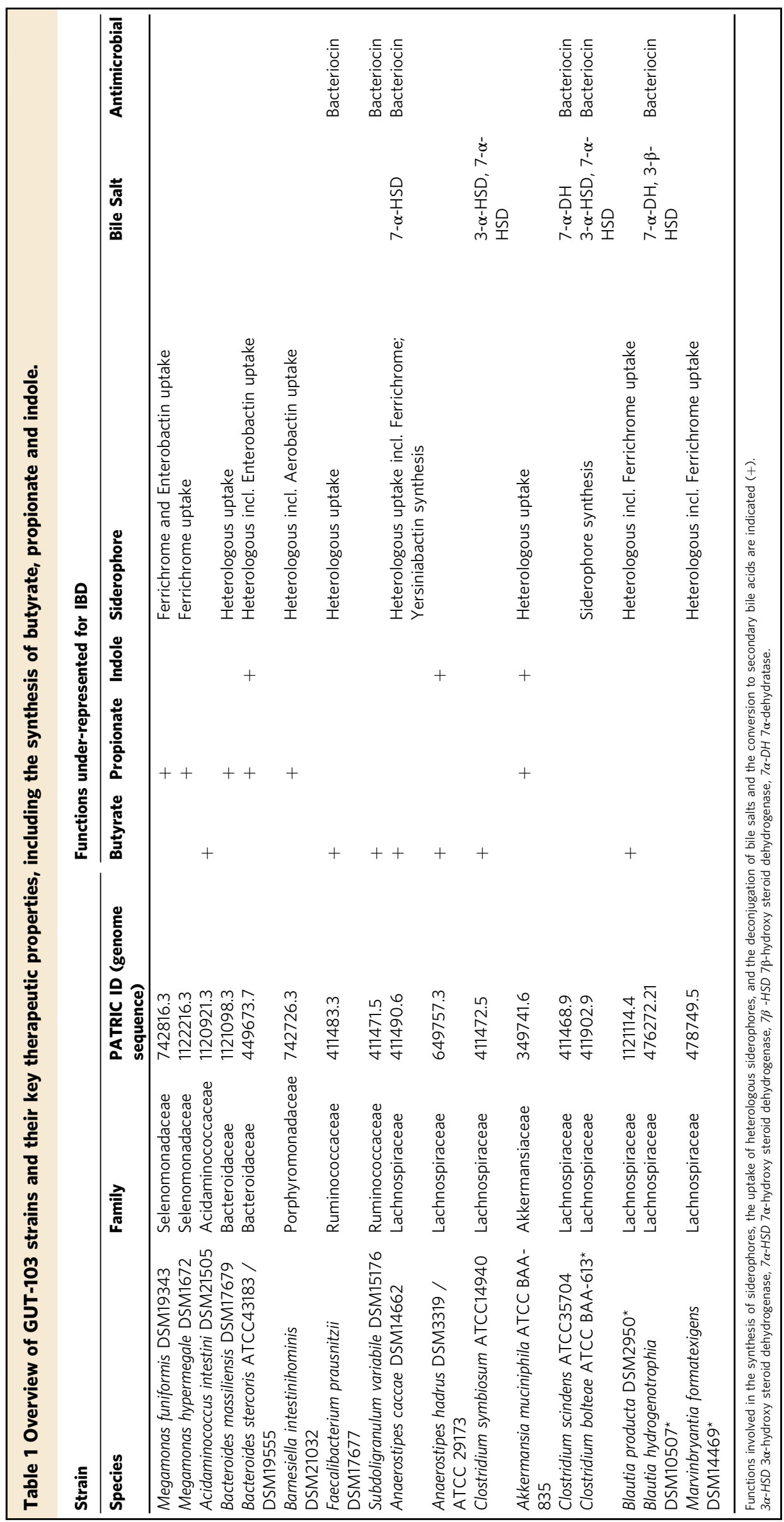


a

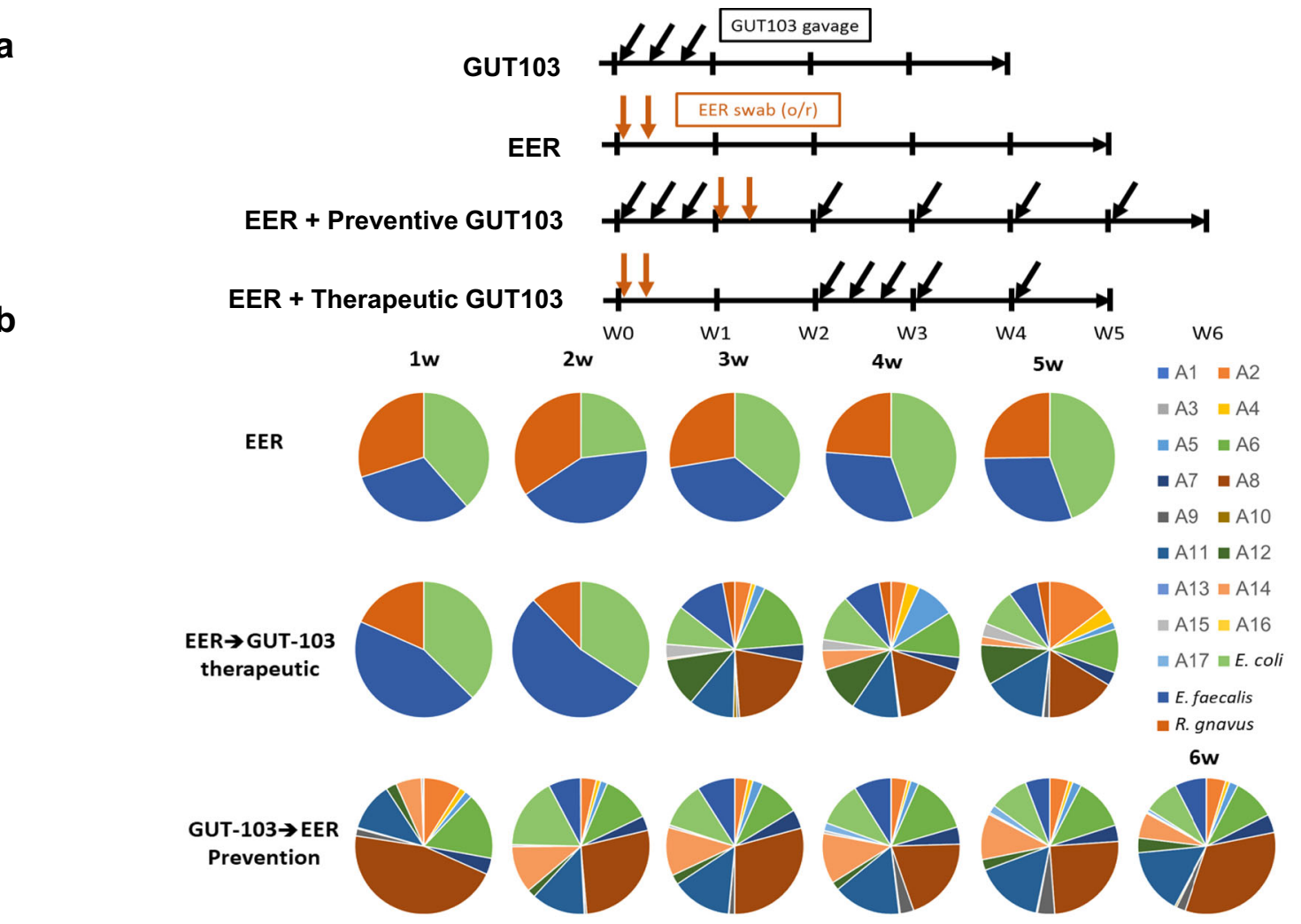

b

C

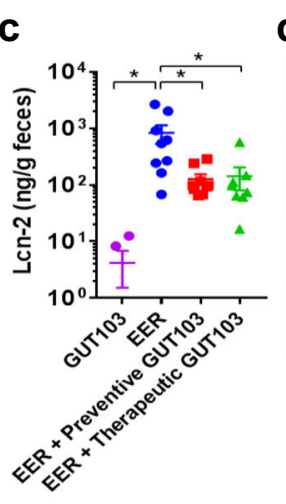

g

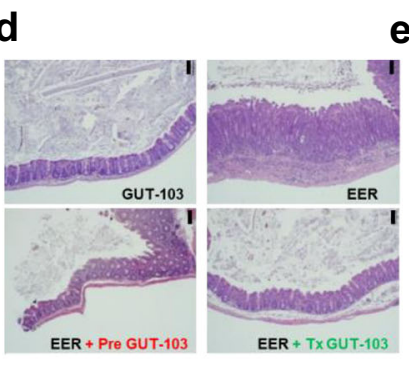

e
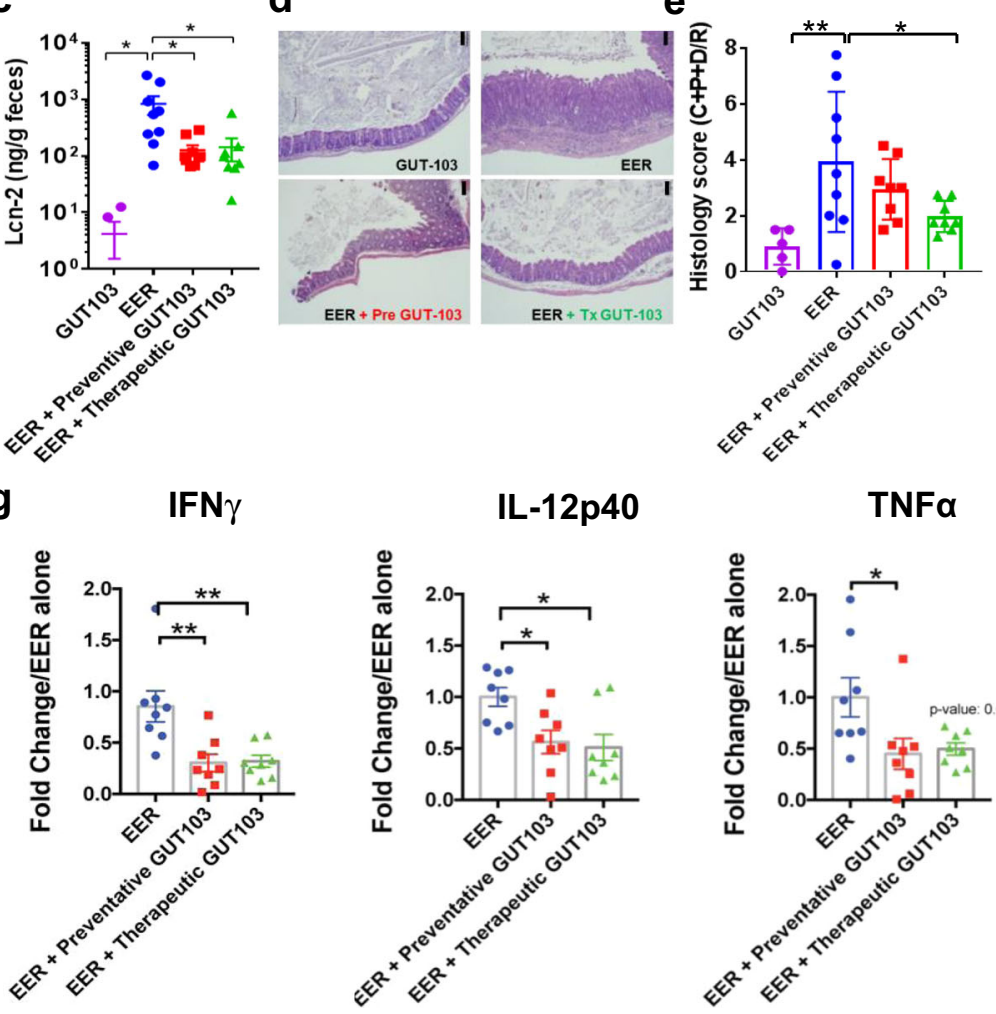

TNFa

$\mathbf{f}$

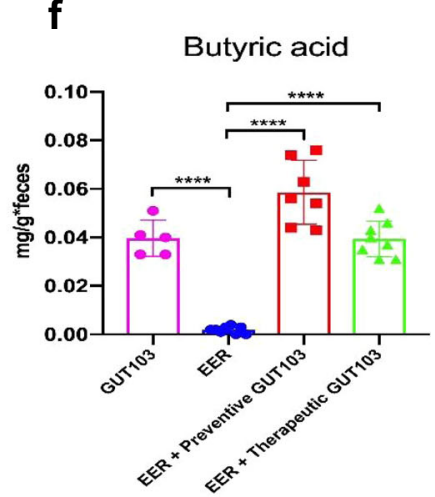

Propionic acid
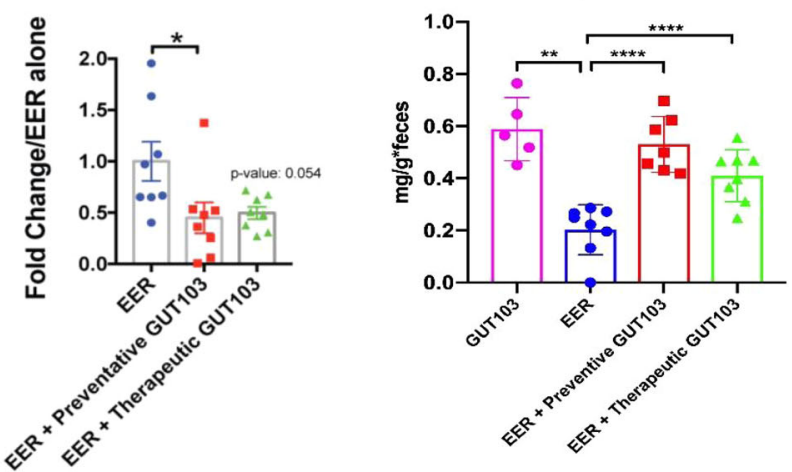

of the individual GUT-108 strains and the GUT-108 consortium under defined conditions and are shown in Supplementary Data 6.

GUT-108 successfully establishes in gnotobiotic mice models. GUT-108 was gavaged into two different GF models, Ill1 $0^{+/ e G F P}$
VertX reporter and $I l 10^{-/-}$mice, with engraftment in both $I l 10^{+} / e G F P$ VertX reporter (Fig. 3a) and $I l 10^{-/-}$mice (Fig. 3b). Colonization levels of individual strains were determined using quantitative PCR. Colonization with GUT-108 did not induce inflammation as documented by representative distal colonic photomicrographs of H\&E-stained tissue (Fig. 3c) and blinded 


\begin{abstract}
Fig. 1 Effect of gavage with GUT-103 on the gut microbiome community composition, lipocalin-2 levels, histology of the gut epithelium, SCFA synthesis and tissue cytokine expression of $1110^{-/-}$mice where experimental colitis was induced by inoculation with Escherichia coli LF82 (E), Enterococcus faecalis OG1RF (E) and Ruminococcus gnavus ATCC29149 (R). a Schematic overview of experimental design. Gnotobiotic $/ 110^{-/}-$mice were inoculated with GUT-103 ( $N=5$ ), EER $(N=9)$, GUT-103 plus EER (preventive protocol) ( $N-7)$, and EER plus GUT-103 (therapeutic protocol) ( $N=7$ ). After 5 or 6 weeks, animals were killed and tissue samples were collected for analysis; b Composition of the microbiome in fecal pellets of gnotobiotic 129 $1 / 10^{-/-}$mice inoculated with EER, GUT-103 plus EER (preventive protocol), and EER plus GUT-103 (therapeutic protocol) determined by qPCR. I/10-/mice were inoculated via gavage with GUT-103 $\left(2 \times 10^{+7} \mathrm{cfu} / \mathrm{strain}\right)$ or EER $\left(2 \times 10^{+7} \mathrm{cfu} / \mathrm{strain}\right)$. The composition of the gut microbiome was determined on fecal pellets or after sacrifice on cecal contents using QPCR with species-specific primers. The average community composition (pie diagrams) for seven animals per treatment are presented. Strain legend: A1: Megamonas hypermegale DSM1672; A2 Bacteroides stercoris DSM19555; A3: Anaerostipes hadrus DSM3319; A4: Clostridium symbiosum ATCC14940; A5: Clostridium boltea ATCC BAA-613; A6: Blautia producta DSM2950; A7: Clostridium scindens ATCC35704; A8: Akkermansia muciniphila ATCC BAA-835; A9: Megamonas funiformis DSM19343; A10: Acidaminococcus intestini DSM21505; A11: Bacteroides massiliensis DSM17679; A12: Barnesiella intestinihominis DSM21032; A13: Faecalibacterium prausnitzii DSM17677; A14: Subdoligranulum variabile DSM15176; A15: Anaerostipes caccae DSM14662; A16: Blautia hydrogenotrophica DSM10507; A17: Marvinbryantia formatexigens DSM14469. c Cecal content lipocalin-2 levels at time of sacrifice from GUT-103 group $(N=5)$, EER group $(N=9)$, GUT-103 plus EER group $(N=9)$, and EER plus GUT-103 group $(N=$ 8), Mann-Whitney unpaired two-tailed t-test. Mean $\pm S E$, ${ }^{\star} P<0.05$. d Representative histology pictures of H\&E-stained cecum tissue $(\times 100)$. The bar (upper right corner) in the pictures indicates $100 \mu \mathrm{m}$ from GUT-103 group (experiment was repeated five times independently with similar results), EER group (experiment was repeated nine times independently with similar results), GUT-103 plus EER group (experiment was repeated eight times independently with similar results), and EER plus GUT-103 group (experiment was repeated eight times independently with similar results). e Histology scores of GUT-103 group $(N=5)$, EER group $(N=9)$, GUT-103 plus EER group $(N=8)$, and EER plus GUT-103 group $(N=8)$. Combined score of cecum, proximal colon, and distal colon/rectum. 1-way ANOVA, Mean $\pm \mathrm{SE},{ }^{\star} P<0.05,{ }^{\star \star} P<0.01$. f Fecal metabolite profiles for butyrate and propionate in $/ 110^{-} /-$ mice inoculated with GUT-103 $(N=5)$, GUT-103 plus EER (preventive protocol, $N=8$ ), and EER plus GUT-103 (therapeutic protocol, $N=8$ ) compared to EER $(N=8)$. Mann-Whitney unpaired two-tailed $t$-test. $\pm S E,{ }^{\star \star} P<0.01,{ }^{\star \star \star \star} P<0.001 . \mathbf{g}$ Cecal tissue levels of cytokine mRNA expression of GUT-103 ( $N=$ 5), EER $(N=8)$, GUT-103 plus EER (preventive protocol, $N=8$ ), and EER plus GUT-103 (therapeutic protocol, $N=8$ ). Samples were collected at necropsy. One-way ANOVA, Mean $\pm \mathrm{SE},{ }^{\star} P<0.05,{ }^{\star}{ }^{*} P<0.01$. Source data and calculated $P$-values are provided as a Source Data file.
\end{abstract}

histologic scores (Fig. 3d). Importantly, GUT-108 colonization promoted a healthy gut microbiome with key functionalities for SCFA, especially synthesis of acetate, butyrate and propionate, and IAA synthesis (Fig. 3e). Four GUT-108 strains, Clostridium butyricum GGCC_0151, Intestinimonas butyriciproducens GGCC_0179, Bitterella massiliensis GGCC_0305, and Barnesiella sp. GGCC_0306 were established at levels below 1\% in both $\mathrm{Il10}^{+} / \mathrm{eGFP}^{-}$VertX reporter (Fig. 3a) and $I l 10^{-/-}$mice (Fig. 3b), with Clostridium butyricum GGCC_0151 failing to get established in $I l 10^{+} / e^{-G P}$ VertX reporter mice. However, due to redundancies in metabolic interdependencies and therapeutic functions, low luminal concentrations of these strains did not prevent restoring key metabolic phenotypes of a healthy gut microbiome. The ability of the GUT-108 consortium to stimulate regulatory (protective) IL-10-mediated immune responses was determined by comparing the type and levels of IL-10-producing immune cells in $I l 10^{+} /$GFP $\operatorname{VertX}$ reporter mice. Two weeks after being introduced, GUT-108 stimulated higher numbers of colonic LP IL-10-producing CD4 ${ }^{+} \mathrm{T}$ cells, B cells, and dendritic cells (DC) and increased numbers and percentages of regulatory $\mathrm{T}$ cells, including inducible Tregs (IL-10 ${ }^{+}$RoR $\gamma \mathrm{T}^{+}$FoxP3 ${ }^{+} \mathrm{CD} 4^{+}$cells and $\mathrm{IL}-10^{+} \mathrm{FoxP}^{+} \mathrm{CD}^{+}$cells) (Fig. $3 \mathrm{f}$ ). Although showing an upward trend, no statistical difference was observed for FoxP3 ${ }^{\text {neg }}$ IL-10 $+\mathrm{T}$ cells $\left(\mathrm{TR}_{1}\right)$.

GUT-108 reverses gut microbiome dysbiosis, restores functionality. Therapeutic application of GUT-108 was tested in a newly developed experimental colitis model using GF $1 l 10^{-/-}$ mice (129SvEv background) humanized with a fecal transplant from a healthy human donor (Fig. 4a). Experimental colitis was induced by inoculating the GF $I l 10^{-/-}$mice with stool from the healthy donor that was previously verified to induce aggressive colitis in ex-GF $I l 10^{-/-}$mice $^{24}$. Over time the normal microbiota from a healthy donor are altered in an inflammatory environment ${ }^{21,25,26}$. We documented in Fig. $3 c$ and Fig. 3d that gnotobiotic $\mathrm{Il}_{10} \mathrm{I}^{-}$mice selectively colonized with the GUT-108 strains did not develop colitis. The difference in outcomes of colitis in $I l 10^{-/-}$mice colonized with the complex microbiota of the healthy human stool versus protection with GUT-108 is likely because GUT-108 contains only protective, commensal bacteria, in contrast to the presence of pathobiont strains present at low levels in healthy human stool, but which expand in a proinflammatory environment. Therapeutic application of GUT-108 beginning 2 weeks after human fecal colonization changed the community composition (Supplementary Fig. 4) and reduced the expansion of opportunistic pathogenic bacteria belonging to the Enterobacteriaceae family that occurred in mice receiving PBS (Supplementary Table 3). Metagenome analysis showed that treatment with GUT-108 resulted in engraftment of all GUT-108 strains except Clostridium scindens GGCC_0168, restoring a gut microbiome that promoted the abundance of beneficial resident Clostridium (Clusters IV and XIVa) strains not part of the GUT108 consortium. Compared to PBS therapy and consistent with the GUT-103 therapeutic results, GUT-108 decreased by $90 \%$ the abundance of Enterobacteriaceae, including Klebsiella pneumoniae, Salmonella enterica, Escherichia coli, and Shigella species, from $5 \%$ to below $0.5 \%$ (Fig. $4 \mathrm{~b}$ ).

The progression of colitis in humanized $1 / 10^{-/-}$mice was evaluated by serial fecal lipocalin-2 (Fig. 4c) after human fecal gavage. Two weeks after human fecal inoculation, elevated levels of fecal lipocalin-2 were observed (compared to basal lipocalin-2 levels of $\sim 3 \mathrm{ng} / \mathrm{g}$ feces material), indicating the onset of moderate to severe colitis. Therapeutic application of GUT108 at 2 weeks after human fecal transplantation decreased inflammation compared to the PBS treatment as measured by fecal lipocalin-2 (Fig. 4c) and blinded histologic scoring (Fig. 4d). GUT-108 decreased histologic inflammation in the cecum and distal colon, as well as the summed colonic score, which we used as an overall inflammatory index $20,21,27$. Representative photomicrographs of the distal colon demonstrated decreased inflammation following GUT-108 therapy with reduced mononuclear cell infiltration in the LP and submucosa, mucosal thickening and crypt hyperplasia (Fig. 4e). Metabolite analysis confirmed that the therapeutic application of GUT-108 restored a physiologically healthy gut microbiome that produced increased levels of anti-inflammatory bacterial metabolites associated with mucosal homeostasis, including the 

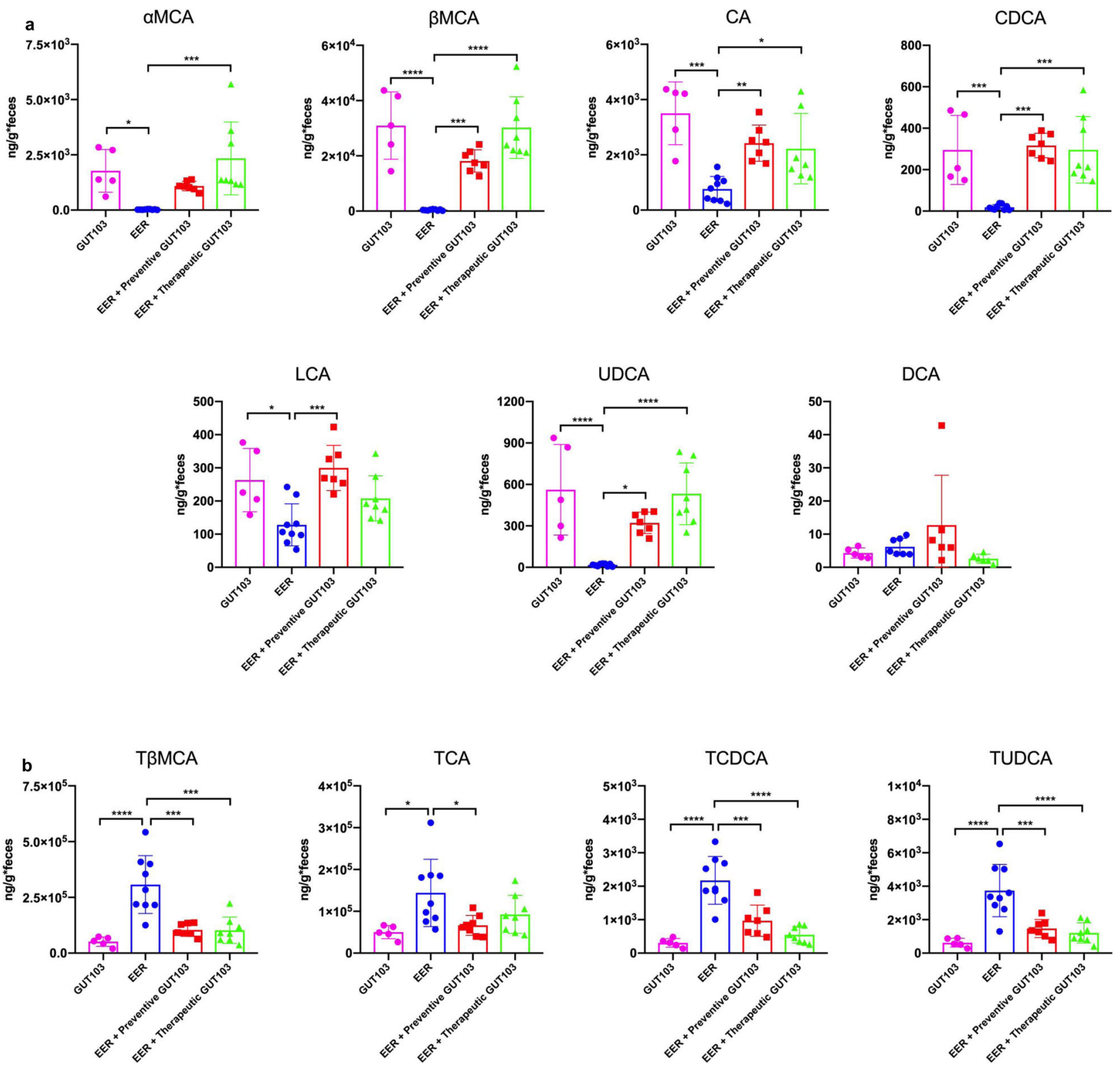

GCA

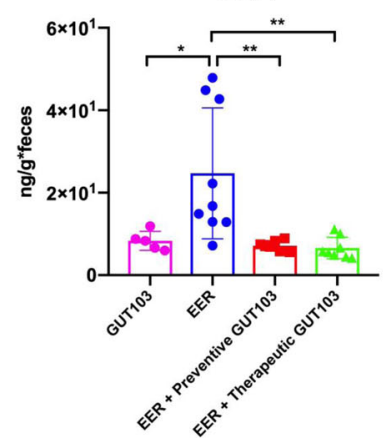

GUDCA

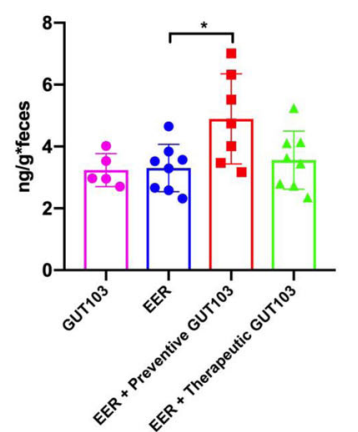

GLCA

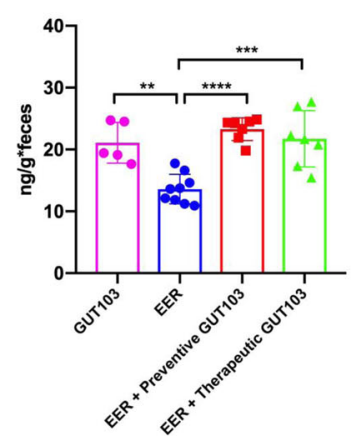

Fig. 2 Levels of unconjugated and conjugated bile acids in the cecal content of $1110^{-/-}$mice at the time of sacrifice where experimental colitis was induced by inoculation with Escherichia coli LF82 (E), Enterococcus faecalis OG1RF (E), and Ruminococcus gnavus ATCC29149 (R). Gnotobiotic I/10-/mice were inoculated with GUT-103 $(N=5)$, EER $(N=9)$, GUT-103 plus EER (preventive protocol) $(N=7)$, and EER plus GUT-103 (therapeutic protocol) $(N=7)$. $1110^{-/-}$mice were inoculated via gavage with GUT-103 $\left(2 \times 10^{+7} \mathrm{cfu} / \mathrm{strain}\right)$ or EER $\left(2 \times 10^{+7} \mathrm{cfu} / \mathrm{strain}\right)$. a Unconjugated bile acids: $\alpha M C A$ $\alpha$-muricholic acid, $\beta$ MCA $\beta$-muricholic acid, CA cholic acid, CDCA chenodeoxycholic acid, LCA lithocholic acid, UDCA ursodeoxycholic acid, DCA deoxycholic acid. b Conjugated bile acids: T $\beta$ MCA taurine-conjugated $\beta$-muricholic acid, TCA taurocholic acid, TCDCA taurochenodeoxycholic acid, TUDCA tauroursodeoxycholic acid, GUDCA glycoursodeoxycholic acid, GCA glycine-conjugated cholic acid, GLCA glycine-conjugated lithocholic acid 1e-way ANOVA, Mean \pm SE, ${ }^{\star} P<0.05,{ }^{\star \star} P<0.01,{ }^{\star \star \star} P<0.005,{ }^{\star \star \star \star} P<0.001$. Source data and calculated $P$-values are provided as a Source Data file. 


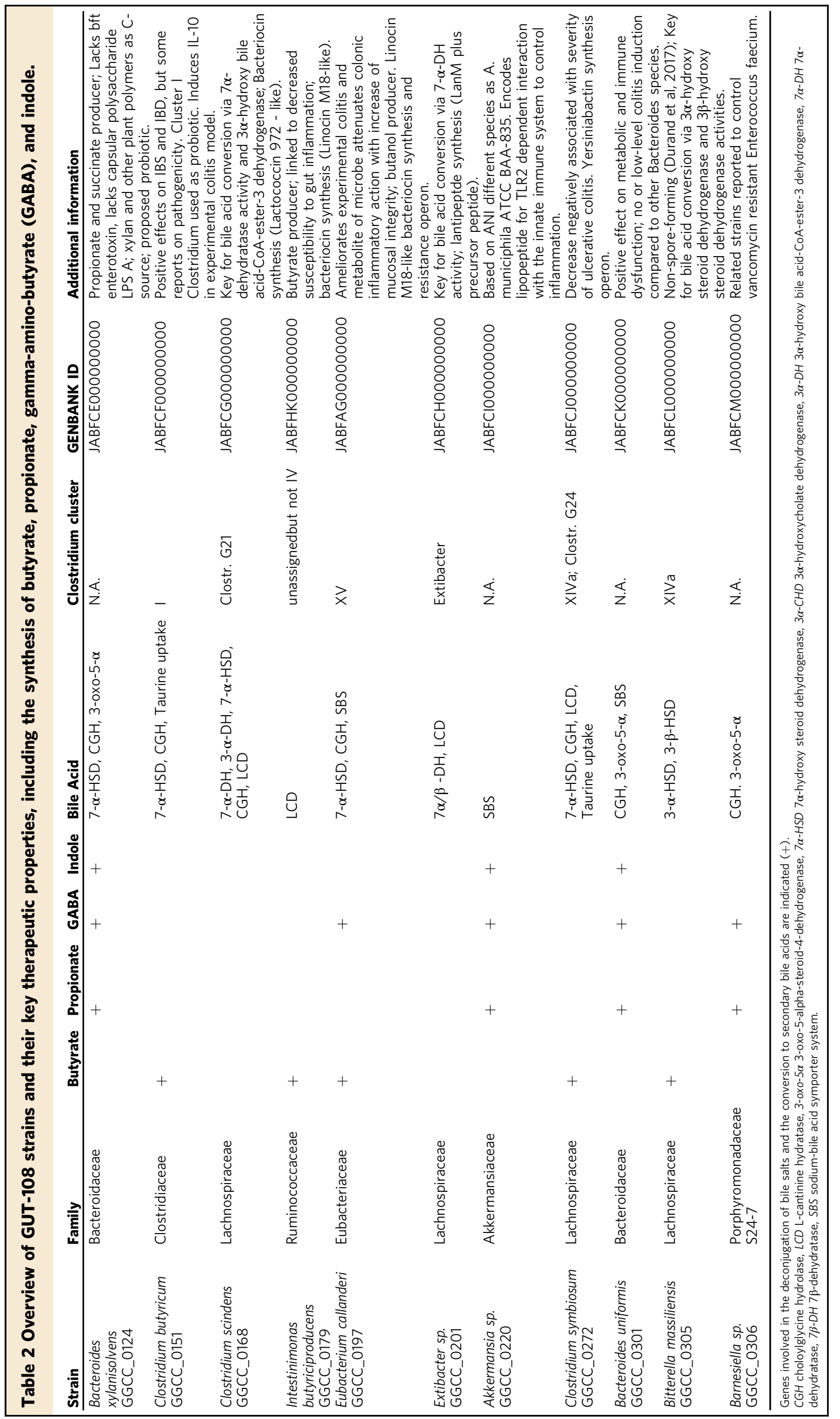



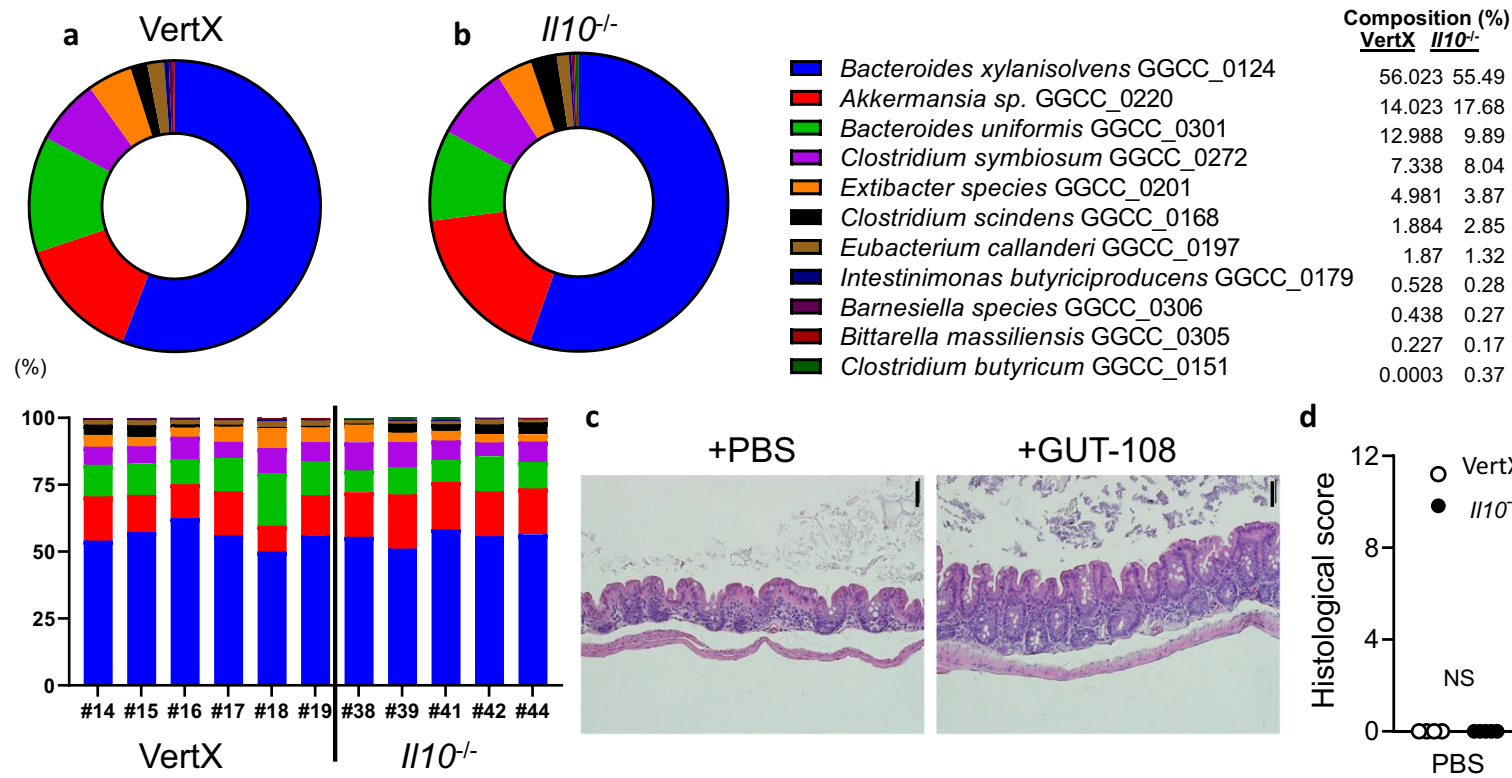

d

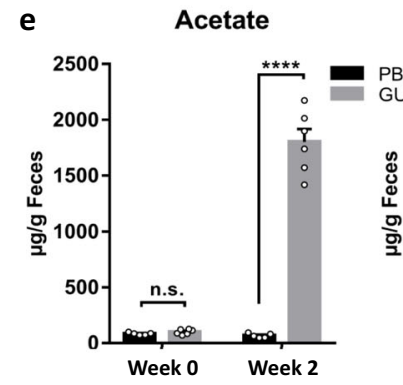

Butyrate

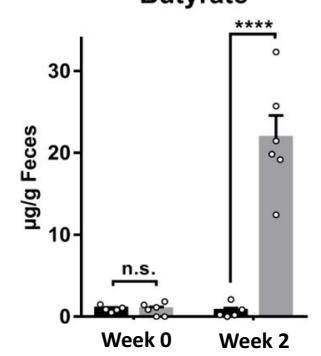

Propionate

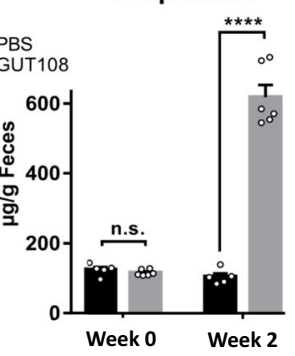

IAA

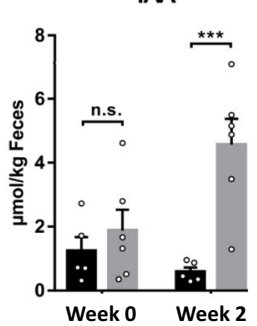

f

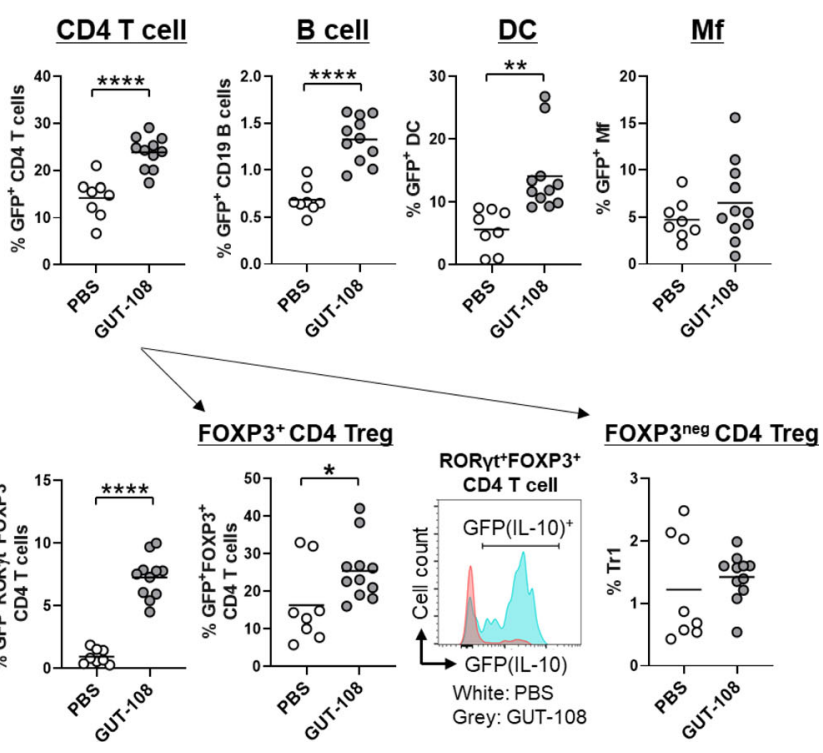

Fig. 3 Effect on the gut microbiome community composition, colonic histology, and fecal metabolomics of gnotobiotic mice inoculated with GUT-108 or treated with PBS for 2 weeks. $1110^{+} / \mathrm{eGFP}$ VertX reporter mice (a) or $1110^{-/-}$mice (b) were inoculated via gavage with GUT-108 (2 $\times 10^{+7}$ cfu/ strain), and after 2 weeks community composition of the gut microbiome was determined on cecal contents using qPCR with species-specific primers. The average community composition (circle diagram) as well as the individual community composition for six $\| 10^{+} / \mathrm{e}^{\mathrm{GFP}} \mathrm{VertX}$ reporter mice and five $1110^{-/-}$mice are presented. The lower detection limit for $\mathrm{GPCR}$ was $>0.0001 \%$. c Representative distal colonic photomicrographs of H\&E-stained tissue showing the lack of colonic inflammation two weeks after inoculation of gnotobiotic $1 / 10^{-/-}$mice with GUT-108 or PBS. The experiment was repeated independently with similar results for each animal in the GUT-108 $(N=11)$ or PBS $(N=8)$ group. The bar (upper right corner) in the pictures indicates $100 \mu \mathrm{m}$. d Histological scoring showing the lack of colonic inflammation 2 weeks after inoculation of gnotobiotic I/10+/eGFP $V$ ertX reporter mice or $1110^{-/-}$mice with GUT-108: combined score of cecum, proximal colon, and distal colon/rectum. Two-Way ANOVA with Sidak's multiple comparison test. NS not significant. $N=5-6 /$ group. e Metabolite analysis of fecal material from $/ 110^{-/-}$mice inoculated with GUT-108 to confirm the successful restoration of bacterial synthesis of acetate, propionate, butyrate and IAA. Two-Way ANOVA with Sidak's multiple comparison test. Bar indicates mean \pm SE. ${ }^{\star \star \star} P<0.001,{ }^{\star \star \star \star} P<0.0005$, N.S. not significant. $N=8$ /group or $N=11$ /group, for PBS and GUT-108 treatment, respectively. f Colonization of gnotobiotic $1110^{+} /$eGFP reporter mice with GUT-108 for 2 weeks induced IL-10-producing T and B cells, dendritic cells (DC) and macrophages (Mf) (top panel) and different types of IL-10+ regulatory T cells (lower panel). GUT-108 refers to II10+ $/$ eGFP $^{2}$ mice treated with GUT-108 $(N=11)$; PBS refers to $1110^{+} /{ }^{e G F P}$ mice treated with PBS buffer $(N=8)$. Dots represent individual mouse results. Bars indicate mean. Mann-Whitney unpaired two-tailed $t$-test. Mean \pm SE. ${ }^{\star} P<0.05 ;{ }^{\star \star} P<0.01 ;{ }^{\star \star \star} P<0.005 ;{ }^{\star \star \star \star} P<0.001 . N=8$ for PBS, $N=11$ for GUT-108. Source data and calculated $P$-values are provided as a Source Data file. 

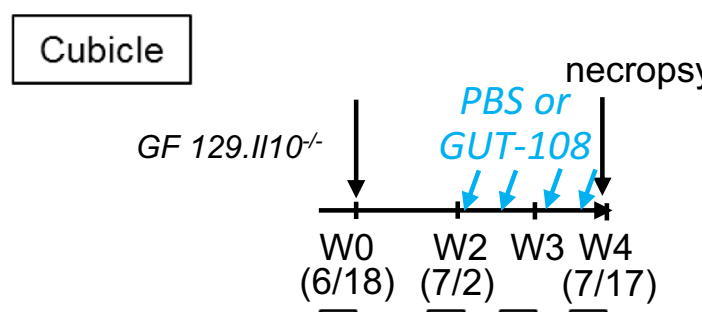

GUT-108 gavage: $2 \times 10^{7}$ bacteria/strain/mouse

$\mathrm{F} F \mathrm{~F}$ b

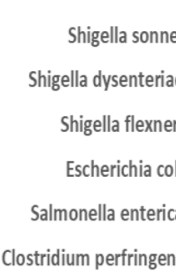

Klebsiella pneumoniae *

Barnesiella sp. GGCC_306

Bacteroides uniformis GGCC_301

Clostridiales bacterium VE202-27*

Clostridium butyricum GGCC_151

Akkermansia sp. GGCC_220

Intestinimonas butyriciproducens GGCC_179

Bacteroides xylanisolvens GGCC_124 *

Lachnoclostridium sp. An14*

Adlercreutzia equolifaciens *

Bittarella massiliensis GGCC_305 *

Clostridium symbiosum GGCC_272*

Eubacterium callanderi GGCC_197*

Dorea sp. D27*

Extibacter sp. GGCC_201 *

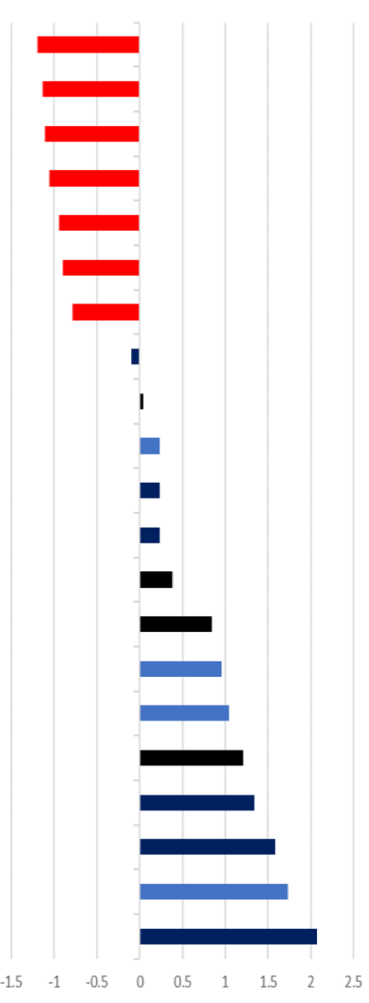

d

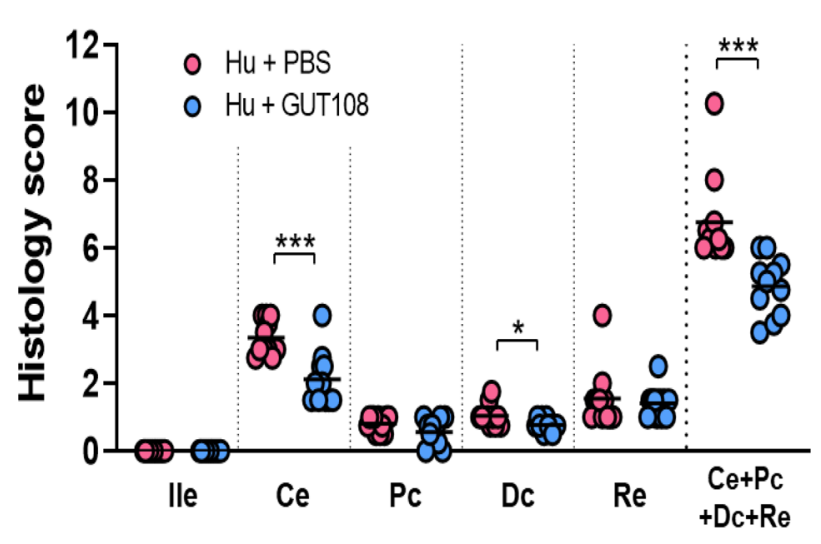

e

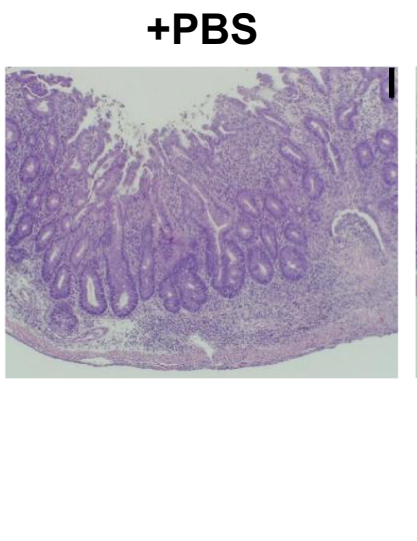

+GUT-108

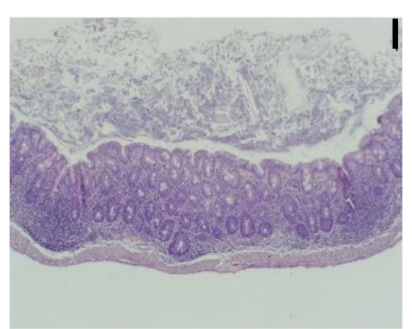

f

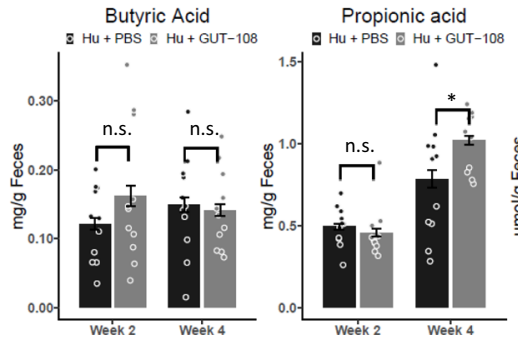

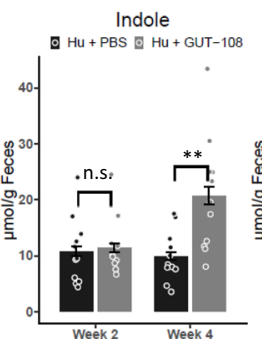
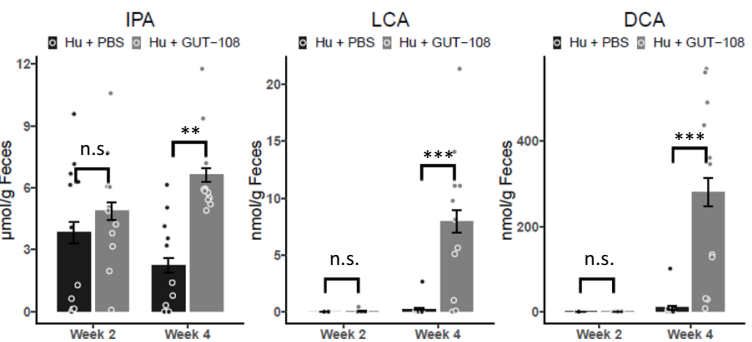

SCFA propionate and synthesis of indole and IPA (Fig. 4f). Likewise, therapeutic application of GUT-108 significantly increased levels of the secondary bile acids LCA and DCA (Fig. 4f), a key objective in the rational design of GUT-108. In contrast to propionate, treatment did not change butyrate levels, consistent with the findings of the selectively colonized gnotobiotic mouse models, where stool butyrate levels were 30fold lower than those for propionate (Fig. 3e).
Therapeutic application of GUT-108 reduces inflammatory cytokines. Reduced severity of inflammation and improved mucosal immune homeostasis after therapeutic application of GUT-108 was characterized by decreased levels of colonic LP effector $\mathrm{CD}^{+}{ }^{+} \mathrm{T}$ cells, including Th1 and Th17 cells that produce the inflammatory cytokines IFN $\gamma$ and IL-17 $\alpha$, respectively, or both IFN $\gamma$ and IL-17a (Fig. 5a); reduced distal colonic expression levels of innate and Th1 and Th17 pathway inflammatory cytokines, 
Fig. 4 GUT-108 strains decreased colitis in II10 ${ }^{-/}$mice colonized with human fecal microbiota. a Schematic overview of experimental design. On day 1 , mice were inoculated with a human stool previously verified to induce aggressive colitis in gnotobiotic $1 / 10^{-/-}$mice. Therapeutic application of GUT-108 or PBS control started after 2 weeks. After 4 weeks necropsies were performed, and tissue samples were collected for analysis. b Bacterial species whose abundance significantly changed in the gut microbiome of mice that received therapeutic application of GUT-108 compared to PBS control mice. Differences in relative species abundance between the two treatments is expressed on a Log10 scale. Strains with decreased (red) or increased (blue) relative abundance are indicated. GUT-108 strains are indicated in black. ${ }^{\star} P<0.05$ value using the two-sided Benjamini-Hochberg corrected Wilcoxon test. c Serial fecal lipocalin-2 levels were measured using ELISA after 2 (before start of GUT-108 or PBS therapy), 3 and 4 weeks. Dots indicate individual mice data. Mann-Whitney unpaired two-tailed $t$-test. Bars indicate Mean $\pm \mathrm{SE},{ }^{\star} P<0.05,{ }^{\star \star \star} P<0.001, N$.S. indicates not significant. $N=11 /$ group. $\mathbf{d}$ Blinded histologic scoring assessed the level of inflammation for different parts of the intestine, including the distal ileum (lle), cecum (Ce), proximal- (Pc) and distal- (Dc) colon, rectum (Re), and combined ( $C e+P c+D c+R e)$. Dots indicate individual mice data. Mann-Whitney unpaired two-tailed $t$-test. Bars indicate Mean \pm SE. Unpaired two-tailed $t$-test was performed on the data. ${ }^{\star} P<0.05,{ }^{\star \star \star} P<0.001, N S$ not significant. $N=11 /$ group. e Representative distal colonic photomicrographs of H\&E-stained tissue showing colonic tissues 2 weeks after starting therapeutic treatment of humanized $/ 110^{-1-}$ mice with GUT-108 or PBS. The experiment was repeated independently with similar results for each animal in the GUT-108 $(N=11)$ or PBS (N=11) group. The bar in the picture indicates $100 \mu \mathrm{m} ; \mathrm{Hu}+$ GUT-108 refers to humanized $/ 110^{-/-}$mice treated with GUT-108; Hu + PBS refers to humanized $/ 110^{-1} /-$ mice that received PBS as a placebo control. $\mathbf{f}$ Induction of protective fecal metabolites by therapeutic GUT-108 treatment beginning at 2 weeks after the induction of inflammation by human stool. Week 4 represents 2 weeks of therapeutic treatment. Hu+GUT-108 refers to humanized II10-/- mice treated with GUT108; Hu + PBS refers to humanized $1110^{-/-}$mice that received PBS buffer. DCA deoxycholic acid, LCA lithocholic acid, IPA indole-3-propionic acid. Twoway ANOVA and adjusted $P$-values were calculated by the multiple comparisons test. Bar indicates Mean \pm SE. N.S. not significant; ${ }^{*}$ : adjusted $P<0.05$; ${ }^{\star *}$ : adjusted $P<0.01 ;{ }^{\star \star *}$ : adjusted $P<0.001$. Source data and calculated $P$-values are provided as a Source Data file.

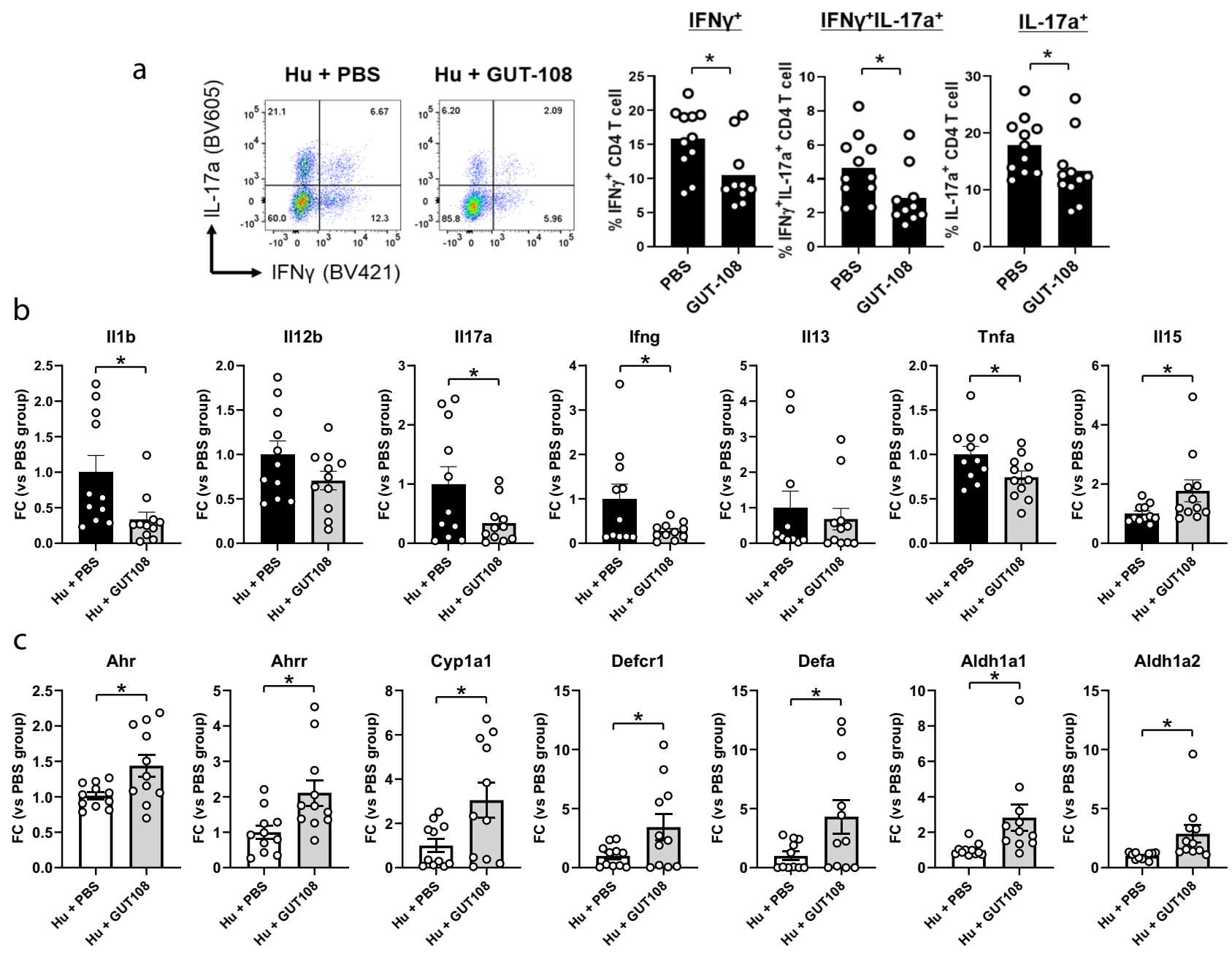

Fig. 5 Effects of treatment with GUT-108 on the levels of inflammatory biomarkers. a Colonic lamina propria effector CD4 $+\mathrm{T}$ cells, including Th1 and Th17 cells that produce IFN $\gamma$ and/or IL-17 $\alpha$. The mRNA expression levels of $\mathbf{b}$ inflammatory cytokines and $\mathbf{c}$ functions important for mucosal homeostasis in the distal colon. Expression levels were determined by RT-Q-PCR and presented as fold change (FC) of expression after therapeutic application of GUT-108 compared to expression after PBS treatment. Hu + GUT-108 refers to humanized $1110^{-/-}$mice treated with GUT-108 2 weeks after fecal colonization; $\mathrm{Hu}+\mathrm{PBS}$ refers to humanized $1 / 10^{-/-}$mice that received PBS as a placebo control. AhR aryl hydrocarbon receptor gene, AhrR aryl hydrocarbon receptor repressor gene, Cyp1A1 Cytochrome P450 Family 1 Subfamily A Member 1gene, DefCR1 and DefA genes defensins, Aldh1A1 and Aldh1A2 genes aldehyde dehydrogenases. Bar indicates Mean \pm SE. Two-way ANOVA and adjusted $P$-value were calculated by the multiple comparisons test. *: adjusted $P<0.05$. $N=11 /$ group. Source data and calculated $P$-values are provided as a Source Data file. 
including IL-1 $\beta$, IL-12p40, IL-13, IL-17a, IFN $\gamma$, and TNFa (Fig. 5b), and increased colonic expression levels of receptors and pathways implicated in mucosal healing (Fig. 5c, Supplementary Fig. 5).

Several protective pathway components that are decreased in IBD were upregulated 2 weeks after application of GUT-108, further supporting its therapeutic role and providing mechanisms for protection (Fig. 5c). Expression of the Ahr pathway genes, including the aryl hydrocarbon receptor (Ahr), the aryl hydrocarbon receptor repressor (AhrR), and the Cytochrome P450 Family 1 Subfamily A Member 1 (Cyp1A1) gene ${ }^{28}$, were significantly upregulated. Protective pathways with increased gene expression levels included the genes for the defensins DefCR1 and DefA, whose altered production is suggested to be integrally involved in IBD pathogenesis ${ }^{29}$, and the genes for aldehyde dehydrogenases Aldh1A1 and Aldh1A2, whose expression is reduced in colonic macrophage and DC subsets of patients with ulcerative colitis regardless of inflammation ${ }^{30}$ (Fig. 5c). Together these results confirm, by multiple readouts, that administration of GUT-108 treats established colitis in humanized mice, out-competes pathobionts, inhibits effector immune responses, and restores homeostatic metabolic and protective immune responses.

\section{Discussion}

Resident bacterial strains of the GUT-103 and GUT-108 consortia, designed to interdependently restore normal function to the inflamed colon, rapidly colonized gnotobiotic mice as well as ex-germ-free humanized mice that had developed a proinflammatory gut microbiome. These GUT-103 and 108colonized mice exhibited the desired functional properties consistent with the rational design of the two consortia.

The two separate rationally designed consortia of intestinal bacterial strains with redundant functionalities that were previously found to be under-represented in the gut microbiome of IBD patients with active disease ${ }^{3}$ were shown to promote homeostatic immune functions and bacterial metabolism and prevent onset or progression of intestinal inflammation. We believe that this approach has the potential to maintain long term remission in a physiologic and safe manner, which should be tested in a Phase 1 clinical trial.

Testing GUT-103 against EER-induced colitis in gnotobiotic $I l 10^{-/}$mice is clinically relevant to human Crohn's disease because adherent-invasive Escherichia coli and Ruminococcus gnavus are increased in active Crohn's disease $e^{3,31,32}$ and the adherent-invasive $E$. coli strain used was isolated from the ileum of a Crohn's disease patient ${ }^{33}$. GUT-108 showed therapeutic efficacy to reverse established colitis in $1 l 10^{-/-}$humanized mice by intervening 2 weeks after onset of moderate-severe intestinal inflammation, supporting a potential role for GUT-108 to treat active IBD. Moreover, the $I l 10^{-/-}$model of chronic pathobiontdriven T-cell-mediated chronic inflammation is more predictive of therapeutic responses in IBD patients than acute epithelial injury models such as dextran sodium sulfate ${ }^{2}$.

GUT-103 and GUT-108 were designed based on human data showing that protective functions provided by commensal bacteria are under-represented in the gut microbiome of IBD patients. These functions include the synthesis of SCFAs, indole and its derivatives, bile acid deconjugation and conversion, and competition for the critical nutrient iron and the synthesis of antagonistic molecules to control opportunistic pathogens ${ }^{3,34}$. Several animal studies have highlighted the role for SCFAs, especially propionate and butyrate, in regulatory $\mathrm{T}$ cell recruitment and function $8,12,13,34-36$. The recruitment in the colon and extrathymic conditioning of regulatory $\mathrm{T}$ cell response by SCFA make these molecules an important link in the crosstalk between the gut microbiome and the immune system. Therefore, commensal bacteria identified to produce propionate and butyrate were selected in the rational design of GUT-103 (Table 1) and GUT-108 (Table 2).

Several studies have shown the role of indole, a metabolite produced from tryptophan, and its metabolites in reducing attachment of pathogenic $E$. coli to epithelial cells ${ }^{37}$, strengthening the mucosal barrier and mucin stimulating production ${ }^{38}$. Therefore, commensal bacteria identified to produce indole and its derivatives were selected in the rational design of GUT-103 (Table 1) and GUT-108 (Table 2).

An inflammatory gut microbiota can result in the inefficient microbial conversion of bile salts into their primary and secondary bile acids ${ }^{3}$. IBD patients with an unbalanced gut microbiome due to inflammation have lower fecal and circulating concentrations of secondary bile acids and higher conjugated fecal bile acid concentrations than do healthy subjects ${ }^{39}$. Thus, activities essential for the conversion of primary bile acids, specifically the conversion of CA and TCDCA acid via a multistep process that includes 7-alpha-dehydroxylation by 7-alpha dehydratase (7- $\alpha-\mathrm{DH})$ or 7-alpha-hydroxy steroid dehydrogenase (7a-HSD) activity ${ }^{15}$, are included in the strain selection for GUT103 (Table 1) and GUT-108 (Table 2).

Competition for iron helps drive the competitiveness and establishment of microorganisms ${ }^{40}$. Therefore, GUT-103 (Table 1) and GUT-108 (Table 2) include several strains that synthesize one or more siderophores under iron-limiting conditions. Ideally, these siderophores are insensitive to inhibition by Lipocalin-2, a peptide that inhibits specific siderophores and their uptake, and is a major colonic defense system triggered by bacterial infections. Lipocalin-2 levels were increased after induction of inflammation in $I l 10^{-/-}$mice colonized with the EER consortium (Fig. 1c) or human fecal microbiota (Fig. 4c).

Bacteriocins, of which lantibiotics are considered a specific class, have shown great promise as new antibiotics for therapeutic application, as reviewed by Field et al. ${ }^{41}$. Thus, bacteriocin synthesis was included as a key functionality in strain selection as part the rational design process of GUT-103 (Table 1) and GUT108 (Table 2).

The optimized 11-strain GUT-108 consortium was rationally designed to build on the proof-of-concept results obtained with GUT-103. GUT-108 went beyond members of the Clostridium clusters IV and XIVa strains, including Bacteroides and Akkermansia species. Furthermore, based on their genome analysis, strains with undesirable properties including the presence of transferable antibiotic resistances or putative virulence factors were excluded. We also omitted species that are fastidiously anaerobic, such as Faecalibacterium prausnitzii, from the GUT-108 consortium. Compared to GUT-103, GUT-108 strains provide additional redundancy for the synthesis of the protective secondary bile acids LCA and DCA, plus multiple mechanisms to compete with opportunistic pathogenic Enterobacteriaceae including synthesis of the siderophore yersiniabactin, and lantibiotics. In the inflammatory gut environment of humanized $1110^{-/}$mice, beneficial Lachnospiraceae and Ruminococcaceae family members are decreased while opportunistic Enterobacteriaceae are increased ${ }^{42}$, as reported for Crohn's disease patients ${ }^{32,43}$. Therapeutic application of GUT-108 reduced levels of colitogenic Enterobacteriaceae and increased beneficial resident Clostridium (Clusters IV and XIVa) species, especially Lachnospiraceae including Dorea species and Lachnoclostridium species that are not GUT-108 constituents (Fig. 4b). This altered community composition increased cecal luminal propionate concentrations, but not butyrate levels (Fig. 4f). Previous studies demonstrated that butyrate levels are not necessarily an indicator of a healthy gut microbiome, as butyrate synthesis from fermentation of amino acids such as lysine 
can contribute to inflammation under conditions associated with mucosal permeability ${ }^{44}$.

GUT-108 increased expression of Gpr41 and showed an upward trend for Gpr43 in $I l 10^{-/}$mice humanized with a fecal transplant (Supplementary Fig. 5). As previously reported, SCFA produced by gut bacteria stimulate Tregs ${ }^{8}$ with propionate's effect mediated through GPR $43^{36}$. SCFA also mediate the function of GPR41, a key regulator that controls host energy balance ${ }^{45}$.

With its functional redundancy and metabolically interdependent auxotrophies, GUT-108 is designed to engraft and perform under a wide range of conditions. When applied to Il10 ${ }^{-/-}$mice humanized with a fecal transplant, all GUT-108 strains except Clostridium scindens GGCC_0168 were established for at least 2 weeks. Clostridium scindens has been previously described as one of the essential strains necessary to convert primary bile acids into LCA and DCA. However, despite the absence of this strain, the established functional multi-strain network produced secondary bile acids, with Extibacter sp. GGCC_0201 providing the 7a-dehydratase activity required to convert $\mathrm{CA}$ and $\mathrm{CDCA}$ into the therapeutic secondary bile acids DCA and LCA, respectively. Normalizing the intestinal bile acid profile can restore intestinal epithelial stem cell function ${ }^{46}$, and increase colonic ROR $\gamma^{+}$ Treg cell counts that ameliorate host susceptibility to colitis ${ }^{47}$, while LCA stimulates Treg differentiation and inhibits Th17 cells ${ }^{48}$ consistent with GUT-108's ability to restore secondary bile acid metabolism (Fig. 4f) and activate inducible $\mathrm{IL}-10^{+}$ ROR $\gamma$ FoxP3 ${ }^{+} \mathrm{CD}^{+}$Treg cells (Fig. 3f). GUT-108 stimulated regulatory (protective) immunity by increasing numbers of colonic LP IL-10-producing CD4 ${ }^{+} \mathrm{T}$ cells, $\mathrm{B}$ cells and DC and numbers and percentages of regulatory $\mathrm{T}$ cells, including inducible Tregs (IL- $10^{+} \mathrm{ROR} \mathrm{T}^{+}{ }^{+}$FoxP3 ${ }^{+} \mathrm{CD} 4^{+}$cells) and IL$10^{+}$Tregs (Fig. 3f). We further documented the antiinflammatory effects of therapeutic GUT-108 in $\mathrm{IllO}^{-/-}$mice humanized with a fecal transplant by demonstrating that GUT108 decreased IFN- $\gamma^{+}, \mathrm{IL}-17 \alpha^{+}$, and IFN- $\gamma^{+}$IL-17 $\alpha^{+}$synthesizing colonic LP CD4 ${ }^{+} \mathrm{TH}_{1}$ and $\mathrm{TH}_{17}$ cells (Fig. 5a) and reduced expression levels of innate and Th1 and Th17 pathway cytokines, including IL-1 $\beta$, IL-12p40, IL-13, IL-17 $\alpha$, IFN $\gamma$, and TNFa (Fig. 5b). Interestingly, GUT-108 treatment increased expression of IL-15 mRNA, a homeostatic cytokine that controls $\mathrm{T}$ cell inflammatory responses. Exogenous IL-15 treatment decreases IL-17 $\alpha$ expression by Th17 cells in vitro through STAT5 enrichment at the $I L-17$ locus $^{49}$, consistent with the ability of GUT-108 therapy to increase IL-15 gene expression and decrease IL-17a mRNA expression in colonic LP cells (Fig. 5b).

Increased intestinal bacterial metabolism of tryptophan, especially indole and its derivatives IAA and IPA, activates the $A h r$ pathway. AHR acts as a sensor of the microbiota community and, through its established role of modulating immune functions, maintains host-microbe homeostasis ${ }^{50}$. IPA is also a pregnane $\mathrm{X}$ receptor (PXR) agonist mediating its responses through TLR4 ${ }^{51}$. GUT-108 therapy increased both IAA and IPA levels in stool (Fig. 4f) and colonic Ahr gene expression (Fig. 5c). AHR is a critical mediator of anti-inflammatory responses to infection by bacterial pathogens and of the differentiation and function of immune cells including $\mathrm{T}$ cells, innate lymphoid cells, macrophages and $\mathrm{DC}^{28}$. AHR promotes the expression of the anti-inflammatory cytokine IL-10 and inhibits macrophage apoptosis, decreases the expression of inflammatory cytokines (IL- 6 and TNF- $\alpha$ ) and inhibits activation of NF- $\kappa \mathrm{B}$. Therefore, the $A h r$ pathway is critical to protect from excessive inflammatory cytokine expression and septic shock. In addition, $A h r$ pathway activation protects the mucosa during inflammation ${ }^{52}$.
Efficacy of GUT-108 in $1110^{-/-}$mice is mediated by IL-10independent mechanisms. Further insights into these possible protective mechanisms include increased expression of metabolite sensors and mediators (Gpr41, Gpr43, Fxr, Pxr, Pparg, Fgf15, Fgf21) and pathways mediating differentiation of immune cells including Treg and Breg cells (cMaf, Il5, April, Aid, Bcl6) (Supplementary Fig. 5). For example, intestinal epithelial FGF15 is activated by bile acids serving as ligands for the nuclear receptor farnesoid X receptor (FXR). FXR/FGF15 signaling regulates bile acid homeostasis and protects against experimental colitis ${ }^{53}$. APRIL impacts immune regulatory $\mathrm{T}$ cells by stimulating their proliferation and survival, and directly contributing to their immune suppression ${ }^{54}$. Bcl6 induces IL-10 and follicular T helper cells and regulates the balance of innate lymphoid cells subsets ${ }^{55}$. Decreased expression of Nos2 (Supplementary Fig. 5), a part of the Ace2 -Nos2-IFNy biosynthesis gene cascade ${ }^{56}$, could lead to lower levels of ACE2, a key regulator to control intestinal inflammation induced by epithelial damage $\mathrm{e}^{57}$. Certain viruses, including the coronaviruses SARS-CoV-1 and SARS-CoV-2, use the ACE2 protein for infecting respiratory and intestinal epithelial ${ }^{58}$. Therefore, chronic gut inflammation, as seen in type2 diabetes and obesity ${ }^{59}$ might trigger elevated gut epithelial ACE2 levels, and therefore patients suffering from these conditions could be more sensitive for Coronavirus infection and at higher risk for complications, highlighting the importance of therapeutically targeting the pro-inflammatory gut microbiome as the underlying cause of chronic inflammation ${ }^{60}$.

In addition to analyzing fecal material, we measured cecal microbiota and in some cases cecal luminal metabolites to represent bacterial communities and function within the cecum/ colon, since the cecum is one of the sites of most active inflammation (Fig. 4d). Previous rodent studies have shown broadly similar microbial patterns in cecal luminal and fecal samples ${ }^{61}$. Furthermore, we demonstrate similar cecal (Fig. 2) and fecal (Fig. 4f) secondary bile acid (LCA and DCA) responses to GUT103 and GUT-108, respectively.

Although no animal model completely replicates all clinical features of human Crohn's disease or ulcerative colitis, we believe that our use of a human fecal transplant in germ-free mice to initiate chronic $\mathrm{TH}_{1} / \mathrm{TH}_{17}$-mediated colitis and our treatment protocol of administering GUT-103 and 108 to mice with established mild to moderate inflammation replicates IBD as closely as feasible for preclinical studies. Proof of efficacy in human IBD will have to await a clinical trial.

GUT-103 and GUT-108 combine multiple modes of action to treat the upstream causes of inflammation by correcting the abnormal microbiome environment, activating various IL-10 synthesizing immune cells, lowering inflammatory responses, and restoring bacterial metabolic profiles to levels found in stool samples of healthy individuals. These overlapping protective mechanisms are predicted to maintain long term remission of IBD in a physiologic and safe manner, in contrast to most biologicals, which block downstream immune effector responses by neutralizing a single cytokine or molecule and induce immunosuppression that can be associated with increased infection and neoplasms. These integrated protective mechanisms make GUT-108 a promising novel therapy to treat a range of conditions whose pathogenesis is characterized by dysbiosis-mediated chronic intestinal inflammation and increased mucosal permeability. Besides IBD this could include graft versus host disease, hepatic encephalopathy, alcoholic liver disease, atherosclerosis, hypertension, obesity, metabolic syndrome, and type- 2 diabetes mellitus.

\section{Methods}

Ethics statement. All animal experiments were performed in compliance with all relevant ethical regulations for animal testing and research as described in the 
National Institutes of Health Guide for the Care and Use of Laboratory Animals. The study received ethical approval by the University of North Carolina-Chapel Hill Institutional Animal Care and Use Committee (18-266.0-B).

The human fecal sample for transplant to mice was collected in compliance with all relevant ethical regulations for work with human participants, and with informed consent from the participant under the approved University of North Carolina Institutional Review Board protocol \#17-1528.

Microbiology techniques. All bacterial strains were grown on LYH-BHI medium (Brain-heart infusion medium supplemented with $0.5 \%$ yeast extract (Difco)), except Bitterella massiliensis GGCC_0305 and Barnesiella sp. GGCC_0306, which were grown on Peptone Yeast Glucose broth (Anaerobe Systems). Strains were cultivated at $37^{\circ} \mathrm{C}$ under strict anaerobic conditions $\left(\mathrm{H}_{2}(5 \%), \mathrm{CO}_{2}(10 \%)\right.$-, and $\mathrm{N}_{2}$ $(85 \%) \mathrm{v} / \mathrm{v} / \mathrm{v})$. After growth, cultures were concentrated at $10^{+9} \mathrm{cfu} / \mathrm{ml}$ in phosphate buffered saline (PBS). Cultures were tested for contamination with aerobic bacteria by streaking on LB medium, followed by incubation at $37^{\circ} \mathrm{C}$ under aerobic conditions. For quality control, a culture was considered pure if no aerobic growth was observed and the sequenced $16 \mathrm{~S}$ rRNA gene of each culture matched the anticipated composition.

DNA sequencing. Bacterial genome sequencing libraries were generated using the ThruPLEX DNA-seq Kit (Rubicon Genomics). Individual strain libraries were combined in equimolar proportions in one pool and sequenced by 125 bp pair end read sequencing on the Illumina HiSeq2500. Onboard image processing and base calling were performed. The sequence data quality score (Q score) was used as a quality control metric with the specification that $\geq 80 \%$ of bases must have a $\mathrm{Q}$ score of $\geq 30$. After trimming, sequencing reads were assembled with SPAdes (version 3.13.0) using default parameters ${ }^{62}$ and annotated using Prokka (version $1.14-\mathrm{dev})^{63}$ and the RAST server ${ }^{64-66}$. Genome sequences for GUT-108 strains were submitted to GenBank (Table 2).

DNA from fecal and cecal material was extracted with AllPrep PowerViral DNA/RNA Kit (Qiagen). Metagenome sequence libraries were constructed using the KAPA Hyper DNA library prep. DNA libraries were multiplexed and loaded on an Illumina HiSeq4000 instrument per manufacturer's instructions. Metagenome sequencing was performed using a $2 \times 150$ paired-end configuration; image analysis and base calling were conducted by the HiSeq Control Software (v2.0.12) on the HiSeq instrument. Sequencing reads were trimmed, and mouse reads were filtered out using Trimmomatic (version v0.39) ${ }^{67}$. Species abundance was determined directly on the metagenome sequencing reads with Kaiju (version $v 1.7 .2)^{68}$. The raw sequencing reads from the fecal samples have been deposited with links to BioProject accession number PRJNA703330 in the NCBI BioProject database (https://www.ncbi.nlm.nih.gov/bioproject/).

Multi-genome analysis and modeling. Genome sequences for GUT-108 strains or reference strains, downloaded from GenBank files (National Center for Biotechnology Information, NCBI) or Pathosystems Resource Integration Center (PATRIC) ${ }^{17}$ were consistently annotated using Prokka (version 1.14-dev), the RAST server, antiSMASH (version 5.1.1) ${ }^{69}$, and Blast against custom-built protein databases. Models were reconstructed, gapfilled, and Flux Balance Analysis ${ }^{70}$ (FBA) was run using the KBase fbatools module v2.0, which is currently deployed in beta mode in the KBase narrative interface (https://narrative.kbase.us/). Reconstruction was performed using the "Build metabolic model" app; gapfilling was performed using the "Gapfill Metabolic Model" app; all flux balance analysis was performed using the "Run Flux Balance Analysis" app; community models were assembled using the "Merge Metabolic Models into Community Model" app; and community flux balance analysis was performed using the "Run Flux Balance Analysis" app. As a last step, all models were manually curated and refined prior to final analysis. Auxotrophies were determined by reviewing the Pathway table to determine if the compounds were created via a reaction or combination of reactions. ' + ' indicates the compound is produced. "AA" indicates the compound is not produced. Average nucleotide identity was determined via pyani (version $0.2 .7 . \mathrm{dev})^{71}$. The results are presented in Supplementary Data files 1 to 6.

Quantitative (RT)-PCR of tissue RNA. For RNA stabilization of colon tissue, RNAlater (Qiagen) was used. The total RNA extractions were performed with RNeasy Plus Mini kit (Qiagen, MD, USA). cDNA was created with the SensiFAST cDNA synthesis kit (Bioline, TN, USA) by PCR $\left(25^{\circ} \mathrm{C}, 10 \mathrm{~min} ; 42^{\circ} \mathrm{C}, 15 \mathrm{~min} ; 85^{\circ}\right.$ C, 5 min). Quantitative (RT)-PCR was performed with QuantStudio3 (Thermo Fisher Scientific, PA, USA) using SYBR No-ROX reagents (Bioline, TN, USA) with the following PCR setting: $95^{\circ} \mathrm{C}, 2 \mathrm{~min} ; 95^{\circ} \mathrm{C}, 5 \mathrm{sec} ; 40$ cycles of $\left(60^{\circ} \mathrm{C}, 10 \mathrm{sec} ; 72^{\circ}\right.$ C, $20 \mathrm{sec}$ ); melting curve analysis: $95^{\circ} \mathrm{C}, 15 \mathrm{sec} ; 60^{\circ} \mathrm{C}, 15 \mathrm{sec} ; 95^{\circ} \mathrm{C}, 15 \mathrm{sec}$. The data were created by comparative $\mathrm{Ct}$ method $\left(2^{-\Delta \Delta \mathrm{Ct}}\right)$. Each cDNA sample was analyzed in duplicate for quantitative assessment of RNA amplification. Melting curve analysis confirmed the presence of single products with expected melting temperatures. An overview of the primers used is provided in Supplementary Data 7.

Quantitative (RT)-PCR of bacterial DNA. Q-PCR using strain-specific primers against the single copy $R p o B$ gene were used to quantify the composition of the
GUT-103 and GUT-108 consortia after gavage in gnotobiotic mice. RpoB primers were validated to ensure there was no significant cross-reactivity between their target bacterium and other bacteria that were present in the consortia (Supplementary Fig. 6). The strain-specific RpoB primers are provided in Supplementary Data 7. The genomic DNA was extracted from fecal or culture media using AllPrep PowerViral DNA/RNA Kit (Qiagen). Q-PCR were performed with QuantStudio3 (Thermo Fisher Scientific, PA, USA) using SYBR No-ROX reagents (Bioline, TN, USA) and $10 \mathrm{ng}$ of genomic DNA per strain with the following PCR settings: $95^{\circ} \mathrm{C}$ $3 \mathrm{~min} ; 40$ cycles of $\left(95^{\circ} \mathrm{C}, 5 \mathrm{sec} ; 60^{\circ} \mathrm{C}, 20 \mathrm{sec}\right)$; melting curve analysis: $95^{\circ} \mathrm{C}, 15 \mathrm{sec}$; $60^{\circ} \mathrm{C}, 15 \mathrm{sec} ; 95^{\circ} \mathrm{C}, 15 \mathrm{sec}$. The data were created by comparative $\mathrm{Ct}$ method $\left(2^{-\mathrm{Ct}}\right)$. Melting curve analysis confirmed the presence of single products with expected melting temperatures.

Targeted metabolomics. Targeted metabolomics were conducted to determine concentration levels of bile acids, short-chain fatty acids (SCFAs), and tryptophan/ indole metabolites in mouse feces and/or cecal contents. Organic solvents and reagents of LC/MS-grade (Optima $\left.{ }^{\mathrm{TM}}\right)$, including methanol $(\mathrm{MeOH})$, isopropanol (IPA), acetonitrile (ACN), water, and formic acid (FA), were purchased from Fisher Scientific (Waltham, WA, USA). Reagents of analytical grade, including 1propanol (PrOH), pyridine (Py), propyl chloroformate (PCF), alongside semiconductor-grade sodium hydroxide $(\mathrm{NaOH})$, and anhydrous sodium sulfate $\left(\mathrm{Na}_{2} \mathrm{SO}_{4}\right)$, were purchased from Sigma-Aldrich (St. Louis, MO, USA).

For SCFA analysis, authentic reference chemical standards of acetic acid (AA), propionic acid (PA), and butyric acid (BA) were purchased from Sigma-Aldrich (St. Louis, MO, USA); stable isotope-labelling (SIL) d4-AA, d2-PA, and d2-BA were procured from Fisher Scientific (Waltham, MA, USA). Fecal SCFA extraction and measurement methods were performed as previously described with minor modifications ${ }^{72}$. In brief, $\sim 40 \mathrm{mg}$ sample aliquots were extracted on a TissueLyzer (Qiagen, Hilden, Germany) into $0.005 \mathrm{M} \mathrm{NaOH}$ aqueous solution spiked with 50 $\mu \mathrm{g} / \mathrm{mL}$ of $\mathrm{d} 4-\mathrm{AA}, 10 \mu \mathrm{g} / \mathrm{mL}$ of $\mathrm{d} 2-\mathrm{PA}$, and $10 \mu \mathrm{g} / \mathrm{mL}$ of d2-BA, followed by centrifugation at $13,200 \mathrm{rpm}$ for $20 \mathrm{~min}$. The supernatant was transferred to glass tubes and derivatized by sequential addition of water, $\operatorname{PrOH}: \operatorname{Py}(3: 2, v / v)$, and PCF. Upon capping, brief vortexing and two-min sonication was performed. The propyl derivatives of SCFAs were extracted twice with hexane, combined, and transferred to $\mathrm{Na}_{2} \mathrm{SO}_{4}$-containing vials for GC-MS quantitation. Instrumental analysis was performed on an Agilent $7820 \mathrm{~A}$ gas chromatography system coupling to a 5977B mass spectrometric detector (MSD) (Santa Clara, CA, USA), where $1 \mu \mathrm{L}$ of derivatives was injected under 1:10 split mode for electron ionization and separated by a DB- $5 \mathrm{~ms}$ column $(30 \times 0.25 \times 0.25 \mathrm{~mm})$ (Santa Clara, CA, USA) with $1 \mathrm{~mL} /$ min helium flowing through. The GC oven temperature program was set as follows: initial oven 175 temperature held at $50^{\circ} \mathrm{C}$ for $2 \mathrm{~min}$, ramped to $70^{\circ} \mathrm{C}$ by $10^{\circ} \mathrm{C} / \mathrm{min}$, to $85^{\circ} \mathrm{C}$ by $3^{\circ} \mathrm{C} / \mathrm{min}, 176$ to $110^{\circ} \mathrm{C}$ by $5^{\circ} \mathrm{C} / \mathrm{min}$, to $290^{\circ} \mathrm{C}$ by $30^{\circ} \mathrm{C} /$ min, and finally held at $290^{\circ} \mathrm{C}$ for $8 \mathrm{~min}$; the MSD was operated at full scan mode with scan range of $m / z 30-600$. The relative standard deviations of intra- and interday precision of analysis were below $10 \%$. Lower limits of quantitation (LLOQ) were determined to be $1 \mathrm{ng}$ for AA (on column), $1 \mathrm{ng}$ for PA, and $0.1 \mathrm{ng}$ for BA.

For bile acid analysis, authentic reference standards (in salt forms) of 23 bile acids, including dihydroxycholestanoic acid (DHCA), glycoursodeoxycholic acid (GUDCA), a-hyocholic acid (HCA), taurochenodeoxycholic acid (TCDCA), cholic acid (CA), taurodeoxycholic acid (TDCA), glycodeoxycholic acid (GDCA), chenodeoxycholic acid (CDCA), deoxycholic acid (DCA), lithocholic acid (LCA), ursodeoxycholic acid (UDCA), tauroursodeoxycholic acid (TUDCA), murideoxycholic acid (MDCA), glycocholic acid (GCA), glycohyocholic acid (GHCA), glycochenodeoxycholic acid (GCDCA), tauro- $\omega$-muricholic acid (TwMCA), tauro- $\alpha$-muricholic acid (TaMCA), tauro- $\beta$-muricholic acid (T $\beta$ MCA), taurocholic acid (TCA), $\alpha$-muricholic acid ( $\alpha$ MCA), $\beta$-muricholic acid ( $\beta$ MCA), $\omega$ muricholic acid ( $\omega \mathrm{MCA}$ ) were purchased from Cayman Chemical (Ann Arbor, MI USA) and Steraloids Inc. (Newport, R.I., USA). Synthetic SIL internal standards, including d4-GCA, d6-GDCA and GDHCA, d4-GLCA, d4-GUDCA, d4-TßMCA d4-CA, d4-TUDCA, d9-TCDCA, d6-TDCA, d4-UDCA, d9-CDCA, d6-DCA, and d4-LCA were purchased from Cambridge Isotope Laboratories, Inc. (Tewksbury, MA, USA). Bile acids in feces and/or cecal content were determined using LC-MS/ MS based on previously published procedures with slight modifications $s^{73,74}$. Briefly, $\sim 10 \mathrm{mg}$ cecal contents were extracted with $\mathrm{ACN}$ and $\mathrm{MeOH}$. Twenty microliter of $1 \mu \mathrm{M}$ internal standard mixture of bile acids was spiked in prior to the extraction. The samples were homogenized on a TissueLyzer (Qiagen, Hilden, Germany) followed by centrifugation at $16,000 \mathrm{rpm}$ for $10 \mathrm{~min}$. Both supernatants were combined, centrifuged at 16,000 rpm for $10 \mathrm{~min}$ again, SpeedVac dried, reconstituted with $\mathrm{MeOH}$, and diluted to $1.2 \mathrm{~mL}$ with $0.1 \% \mathrm{FA}$ in water. The mixture extracts were passed through Biotage ISOLUTE ${ }^{\oplus}$ C18 cartridges $(100 \mathrm{mg} / 1$ $\mathrm{mL}$ ) (Uppsala, Sweden) which had been washed with $\mathrm{MeOH}$ and conditioned with $\mathrm{MeOH}$ :water (with $0.1 \% \mathrm{FA})(1: 5, v / v)$ prior to analysis. Upon completion of sample loading, the cartridges were washed with $\mathrm{MeOH}$ :water (with $0.1 \% \mathrm{FA}$ ) and subject to vacuum drying. The analytes were eluted afterwards with $\mathrm{MeOH}$, SpeedVac dried at $8{ }^{\circ} \mathrm{C}$, and resuspended in $50 \% \mathrm{MeOH}$ upon analysis. The instrumental analysis was conducted on a Vanquish UHPLC system coupling to a TSQ Quantis triple quadrupole mass spectrometer (Thermo Scientific, Waltham, MA, USA) interfacing with an electrospray ionization (ESI) source operated in negative ion mode. Chromatographic separation was carried out on an ACQUITY 
UPLC HSS T3 $(2.1 \times 100 \mathrm{~mm}, 1.8 \mu \mathrm{m})$ column (Milford, MA, USA) maintained at $60{ }^{\circ} \mathrm{C}$. The mobile phases consisted of $0.01 \% \mathrm{FA}$ in water $(\mathrm{A})$ and $0.01 \% \mathrm{FA}$ in ACN (B). The bile acids are eluted at a flow rate of $400 \mu \mathrm{L} / \mathrm{min}$ with a gradient elution of mobile phase starting at $25 \% \mathrm{~B}$, held for $2 \mathrm{~min}$; increased to $40 \% \mathrm{~B}$ in 13 $\mathrm{min}$; increased to $98 \% \mathrm{~B}$ at $20 \mathrm{~min}$, held for $2 \mathrm{~min}$; decreased to $25 \% \mathrm{~B}$ at $22.5 \mathrm{~min}$, and held for $3.5 \mathrm{~min}$, for a total run of $26 \mathrm{~min}$. The source parameters were: spray voltage, $3500 \mathrm{~V}$; sheath gas, 50 arbitrary unit; aux gas, 25 arbitrary unit; sweep gas, 6 arbitrary unit; ion transfer tube temperature, $325^{\circ} \mathrm{C}$; vaporizer temperature, $350^{\circ}$ C. Quantification of bile acids was based on an isotope dilution method using the responses of d4-GCA (for GCA and GHCA), d6-GDCA (for GDCA and GDHCA), d4-GLCA (for GLCA), d4-GUDCA (for GCDCA, GHDCA, and GUDCA), d4-

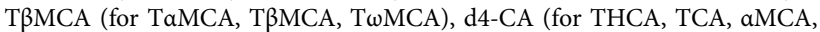
BMCA, $\omega \mathrm{MCA}, \mathrm{CA}$, and HCA), d4-TUDCA (for TUDCA), d9-TCDCA (for TCDCA), d6-TDCA (for TDCA and TDHCA), d4-UDCA (for UDCA), d9-CDCA (for CDCA and MDCA), d6-DCA (for DCA and DHCA), and d4-LCA (for LCA). Calibration curves with at least ten points spanning concentration ranges of $0.1-2000 \mathrm{nM}$ were employed. The data were corrected for differences in the amount of stool analyzed. Regression coefficients $\mathrm{R}^{2}>0.99$ for all calibration curves. LODs for all bile acids were determined as below $0.5 \mathrm{nM}$.

For analysis of tryptophan and indole metabolites, reference chemical standards of tryptophan (Trp), kynurenine (Kyn), indole, indole-3-acetate (IAA), and indole3-propionate (IPA), were purchased from Sigma-Aldrich (St. Louis, MO, USA). SIL internal standards for each compound, except d6-Kyn from Cambridge Isotopes Laboratories (Tewksbury, MA, USA), were obtained from CDN Isotopes (Pointe-Claire, Quebec, Canada). Tryptophan and indole compounds were determined using a LC-MS assay. In brief, $\sim 20 \mathrm{mg}$ feces and/or cecal content aliquots were extracted on a TissueLyzer (Qiagen, Hilden, Germany) with ice-cold $\mathrm{MeOH}$ :water solution $(1: 1, v / v)$ spiked with $500 \mathrm{nM}$ internal standard mixtures. Upon centrifugation at $15,000 \mathrm{rpm}$ for $10 \mathrm{~min}$, the supernatant layer was collected, dried in a Labconco CentriVap vacuum concentrator (Kansas, MO, USA), and resuspended in ACN:water $(2: 98, v / v)$ solution upon instrumental analysis. A Thermo Vanquish UHPLC system coupled to a Q Exactive orbitrap mass spectrometer (Waltham, MA, USA) was used; injected analytes underwent 15-min chromatographic separation on an ACQUITY UPLC HSS T3 column (Waters, Milford, MA) and positive electrospray ionization (ESI), and ions were detected under parallel reaction monitoring (PRM) mode. Internal standard quantitation was performed disparately to determine the concentration levels of the five metabolites, using calibration curves with at least five points and a fitted linearity regression of $\mathrm{R}^{2}>0.99$. LLOQ were determined as 50 femtomole ( $\mathrm{fM}$ ) on column for Kyn and Trp, $100 \mathrm{fM}$ for IAA and IPA, and 0.5 picomole (pmol) for indole. Recovery rates from sample extraction ranged from 80.5 to $100.7 \%$ for the five compounds, and no observable matrix effects were found throughout the analytical procedures.

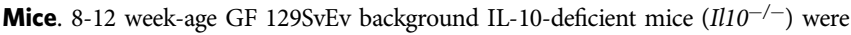
obtained from University of North Carolina National Gnotobiotic Rodent Resource Center $^{20}$. Il10-eGFP-reporter (Il10 $\left.{ }^{+/ \mathrm{eGFP}}\right)$ mice on a C57BL/6 J background mice were originally provided by Dr. C. L. Karp (Global Health, Bill \& Melinda Gates Foundation, USA) and raised in the National Gnotobiotic Rodent Resource Center. Germ-free and gnotobiotic mice were maintained in positive-pressure isolators and housed in separate polycarbonate cages at constant room temperature $\left(22^{\circ} \mathrm{C} \pm 10 \%\right)$, air humidity $(50 \pm 20 \%)$, and a light/dark cycle of $12 \mathrm{~h}$. Mice had free access to food and water. Standard mouse chow (TD2020SX; Teklad Diets, Madison, WI) was sterilized by irradiation at $25 \mathrm{kGy}$. GF mice were colonized with EER or human feces by oral gavage $(200 \mu \mathrm{l})$ and $3-4$ mice/cage were housed within gnotobiotic Trexler isolators (EER/GUT-103 experiments and gnotobiotic GUT-108 experiments) ${ }^{20}$ or in sterilized cages with autoclaved food and water (humanized/GUT-108 experiments). Animal use protocols were approved by the Institutional Animal Core and Use Committee of the University of North Carolina at Chapel Hill.

Inoculation. For treatment $200 \mu \mathrm{l}$ of diluted human donor stool was applied by oral gavage on day 1 to $I l 10^{-/-}$mice. The stool was derived from a single healthy donor, Donor-Y, and was previously found to induce moderate to severe colitis in Ill10 ${ }^{-/-}$mice $^{24}$. One gram stool was diluted 100 -fold with anaerobic PBS, and vigorously mixed for $5 \mathrm{~min}$ under anaerobic conditions. For application of GUT103 and GUT-108, $300 \mu \mathrm{l}$ resuspended strain mixture in anaerobic PBS was applied per mouse by oral gavage. GUT-103 and GUT-108 strains were grown individually, subsequently mixed to equal concentrations ( $\mathrm{cfu} / \mathrm{ml})$, and provided at a dose of $2.0 \times 10^{+7} \mathrm{cfu} / \mathrm{strain}$ in a total volume of $300 \mu \mathrm{l}$. The strain mixture was provided four times via oral gavage on days $15,17,22$, and $25\left(I l 10^{-/-}\right.$mice $)$or on days 1,3 , 8 , and 11 (Illo+leGPP mice).

Fecal collections. Fresh murine feces ( $2-5$ pieces/mouse) were collected and immediately snap-frozen on dry ice and stored at $-80^{\circ} \mathrm{C}$. The human fecal sample used for murine fecal transplant was isolated from a healthy volunteer and stored at $-80^{\circ} \mathrm{C}$.

Cell isolation. Colonic tissues were opened longitudinally, washed twice with $1 \times$ PBS, cut into $1 \mathrm{~cm}$ pieces and incubated with stirrer for 250 r.p.m. in HBSS (Corning) medium containing 2.5\% FBS (Sigma-Aldrich), 1\% penicillin-streptomycin (Gibco), $4 \mathrm{mM}$ EDTA (Corning) and $10 \mathrm{mM}$ dithiothreitol (Sigma) for $20 \mathrm{~min}$ at $37^{\circ} \mathrm{C}$ to remove the epithelial layer. Denuded tissue samples were washed twice with HBSS containing $2.5 \%$ FBS and $1 \%$ penicillin-streptomycin and incubated with a stirrer at 450 r.p.m. in HBSS containing 2.5\% FBS, $0.5 \mathrm{mg} / \mathrm{ml}$ of collagenase (Sigma) for $30 \mathrm{~min}$ at $37^{\circ} \mathrm{C}$. Cell preparations were filtered through $100-\mu \mathrm{m}$ nylon mesh to achieve single-cell suspensions. LP cells were purified using a 40-70\% discontinuous Percoll gradient (GE Healthcare, 2000 r.p.m., 20 min, room temperature) and washed with HBSS

Staining cells for flow cytometry analysis. Single cells were stained for $20 \mathrm{~min}$ at $4{ }^{\circ} \mathrm{C}$ after Fc $\gamma$ RII/III blocking with anti-CD16/CD32 monoclonal antibody. For intracellular staining, cells were restimulated with $50 \mathrm{ng} / \mathrm{ml}$ phorbol 12 -myristate 13-acetate (PMA, Sigma) and $500 \mathrm{ng} / \mathrm{ml}$ ionomycin (Sigma) for $4 \mathrm{~h}$ at room temperature with $1 \mu \mathrm{l} / \mathrm{ml}$ protein transport inhibitor (GolgiStop, BD) during the last $3 \mathrm{~h}$. After washing, cells were first surface stained, then fixed for $5 \mathrm{~min}$ at $37^{\circ} \mathrm{C}$ using PBS containing 4\% paraformaldehyde (Electron Microscopy Sciences) and $0.01 \%$ Tween 20 (Fisher Scientific), permed using PBS containing $0.1 \%$ Triton X100 (MP Biomedicals), 0.5\% BSA (Sigma), $2 \mathrm{mM}$ EDTA (Corning) for $45 \mathrm{~min}$ at room temperature, and stained overnight with indicated antibodies. Flow cytometry was performed on a LSRII flow cytometer with FACSDiva software version 6.0 (BD Biosciences). Singlet live $\mathrm{CD} 45^{+}$cells were analyzed by FlowJo software version 10 (FlowJo, OR, USA) with the following gating strategy: B cell (B220 $\left.{ }^{+} \mathrm{CD} 19^{+}\right), \mathrm{CD}^{+} \mathrm{T}$ cell $\left(\mathrm{TCR} \beta^{+} \mathrm{CD} 3^{+} \mathrm{CD} 4^{+} \mathrm{CD} 8^{\text {neg }}\right)$, macrophage

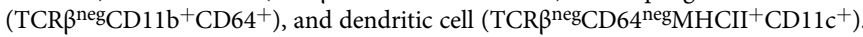
For the GFP-positive gate, GFP-negative colonic LP cells from C57BL/6 wild-type mice were stained with all antibodies used in the experiment as a fluorescenceminus-one control. The flow cytometry gating strategies used to collect the data presented in Fig. $3 \mathrm{f}$ and Fig. 5a are provided in Supplementary Fig. 7 and Supplementary Fig. 8, respectively.

The following antibodies were used for flow cytometry: anti-CD16/CD32 monoclonal antibody (Fc block, BD Biosciences, Cat\# 553141), Live/Dead fixable dead cell stains (APC-Cy7, Invitrogen, Cat\# L10119) at dilution of 1:2400, antiCD45 antibody (Pacific Orange, Invitrogen, Cat\# MCD4530) at dilution of 1:300, anti-B220 antibody (Pacific Blue, Invitrogen, Cat\# 48-0452-82) at dilution of 1:200, anti-CD19 antibody (BV605, Biolegend, Cat\# 115539) at dilution of 1:300, anti-CD4 antibody (eFluor450, eBioscience, Cat\# 48-0041-80) at dilution of 1:200, anti-CD4 antibody (APC, Biolegend, Cat\# 100516) at dilution of 1:400 anti-CD3 antibody (PE-Cy5, eBioscience, Cat\# 15-0031-81) at dilution of 1:300, anti-TCRb antibody (Alexa Fluor 700, BD Biosciences, Cat\# 560705) at dilution of 1:300, anti-CD8 antibody (PE-Cy7, eBioscience, Cat\# 25-0081-81) at dilution of 1:300, anti-CD11b antibody (PE-Cy7, BD Biosciences, Cat\# 552850) at dilution of 1:300, anti-CD11c antibody (Alexa Fluor 700, BD Biosciences, Cat\# 560583) at dilution of 1:400, anti-CD64 antibody (PE, Biolegend, Cat\# 139304) at dilution of 1:300, anti-MHC classII antibody (BV650, Biolegend, Cat\# 107641) at dilution of 1:300, anti-IFNg antibody (BV421, Biolegend, Cat\# 505829) at dilution of 1:300, anti-IL-17a antibody (BV605, Biolegend, Cat\# 506927) at dilution of 1:300, anti-Foxp3 antibody (PE, eBioscience, Cat\# 12-5773-82) at dilution of 1:300, anti-RORgt antibody (Alexa Fluor 647, BD Biosciences, Cat\# 562682 ) at dilution of 1:300, isotype IgG (PE-Cy5, Biolegend, Cat\# 400931) at dilution of 1:300, IgG2 lambda (Alexa Fluor 700, BD Biosciences, Cat\# 557985) at dilution of 1:300, IgG2a (APC, BD Biosciences, Cat\# 554690) at dilution of 1:300, IgG2a (PE-Cy7, Biolegend, Cat\# 400521) at dilution of 1:300, IgG2b (BV650, BD Biosciences, Cat\# 563233) at dilution of 1:300, IgG2a (BV605, Biolegend, Cat\# 400539) at dilution of 1:300, and IgG2b (Pacific Orange, BD Biosciences, Cat\# 553989) at dilution of 1:300.

Histology and scoring. Fixing, staining, and blind histology scoring were described previously ${ }^{20}$.

Statistical analysis. GraphPad Prism (version 8) software was used for statistical analysis. For the metabolite analyses, the two-way ANOVA in Graph Pad Prism 8 software was applied to determine the statistical significance of SCFAs and bile acids among groups. Dunnett's multiple comparisons test was performed to determine whether a specific SCFA or bile acid is significantly different between EER control and other groups. An adjusted $p$-value (calculated by Graph Pad Prism 8 software, version 8.0 .0 ) of $<0.05$ was considered as significant difference. Results were adjusted based on the amount of stool used.

Reporting summary. Further information on research design is available in the Nature Research Reporting Summary linked to this article.

\section{Data availability}

The relevant data are available from the authors upon reasonable request. Metagenome sequencing data that support the findings of this study have been deposited in GenBank with the BioProject ID: PRJNA703330. The genome sequences for the GUT-103 consortium strains were downloaded from PATRIC (https://www.patricbrc.org) with the accession codes: 742816.3; 1122216.3; 1120921.3; 1121098.3; 449673.7; 742726.3; 
$11483.3 ; 411471.5 ; 411490.6 ; 649757.3 ; 411472.5 ; 49741.6 ; 411468.9 ; 411902.9 ; 1121114.4$; $476272.21 ; 478749.5$. The genome sequences for the novel strains used in the GUT-108 consortium that support the findings of this study have been deposited in GenBank with the accession codes: JABFCE000000000, JABFCF000000000, JABFCG000000000, JABFHK000000000, JABFAG000000000, JABFCH000000000, JABFCI000000000, JABFCJ000000000, JABFCK000000000, JABFCL000000000, and JABFCM000000000. Raw data for the targeted metabolomics are available from Metabolomics Workbench repository, https://doi.org/10.21228/M8XM6R, project ID PR001120. The bacterial strains used in the GUT-103 consortium are commercially available from ATCC (https:// www.ATCC.org) and DSMZ (https://www.DSMZ.de). The bacterial strains used in the GUT-108 consortium can be obtained from the corresponding author through a Material Transfer Agreement with the restriction that they can only be used for academic research purposes. Source data are provided with this paper.

Received: 3 June 2020; Accepted: 29 April 2021;

Published online: 28 May 2021

\section{References}

1. de Souza, H. S. \& Fiocchi, C. Immunopathogenesis of IBD: current state of the art. Nat. Rev. Gastroenterol. Hepatol. 13, 13-27 (2016).

2. Sartor, R. B. \& Wu, G. D. Roles for intestinal bacteria, viruses, and fungi in pathogenesis of inflammatory bowel diseases and therapeutic approaches. Gastroenterology 152, 327-339 e324 (2017).

3. Lloyd-Price, J. et al. Multi-omics of the gut microbial ecosystem in inflammatory bowel diseases. Nature 569, 655-662 (2019).

4. Oka, A. \& Sartor, R. B. Microbial-based and microbial-targeted therapies for inflammatory bowel diseases. Dig. Dis. Sci. 65, 757-788 (2020).

5. Eeckhaut V. et al. In 5th Probiotics, Prebiotics and New Foods Congress, Rome, Italy. http://www.probiotics-prebiotics-newfood.org/pdf/5th_Probiotics_ Prebiotics_Newfood.pdf.

6. Van Immerseel, F. et al. Butyric acid-producing anaerobic bacteria as a novel probiotic treatment approach for inflammatory bowel disease. J. Med. Microbiol. 59, 141-143 (2010).

7. Atarashi, K. et al. Treg induction by a rationally selected mixture of Clostridia strains from the human microbiota. Nature 500, 232-236 (2013).

8. Furusawa, Y. et al. Commensal microbe-derived butyrate induces the differentiation of colonic regulatory T cells. Nature 504, 446-450 (2013).

9. Tanoue, T. et al. A defined commensal consortium elicits CD8 T cells and anti-cancer immunity. Nature 565, 600-605 (2019).

10. Administration, U. S. F. D. FDA Safety Alert Regarding the Use of Fecal Microbiota for Transplantation and Risk of Serious Adverse Events Likely due to Transmission of Pathogenic Organisms (U.S. Food \& Drug Administration, 2020).

11. DeFilipp, Z. et al. Drug-resistant E. coli bacteremia transmitted by fecal microbiota transplant. N. Engl. J. Med. 381, 2043-2050 (2019).

12. Michaudel, C. \& Sokol, H. The gut microbiota at the service of immunometabolism. Cell Metab. https://doi.org/10.1016/j.cmet.2020.09.004 (2020).

13. Lavelle, A. \& Sokol, H. Gut microbiota-derived metabolites as key actors in inflammatory bowel disease. Nat. Rev. Gastroenterol. Hepatol. 17, 223-237 (2020).

14. Ridlon, J. M., Kang, D. J. \& Hylemon, P. B. Bile salt biotransformations by human intestinal bacteria. J. Lipid Res. 47, 241-259 (2006).

15. Kang, D. J., Ridlon, J. M., Moore, D. R. 2nd, Barnes, S. \& Hylemon, P. B. Clostridium scindens baiCD and baiH genes encode stereo-specific 7alpha/ 7beta-hydroxy-3-oxo-delta4-cholenoic acid oxidoreductases. Biochim. Biophys. Acta 1781, 16-25 (2008).

16. Tatusova, T. et al. NCBI prokaryotic genome annotation pipeline. Nucleic Acids Res. 44, 6614-6624 (2016).

17. Wattam, A. R. et al. PATRIC, the bacterial bioinformatics database and analysis resource. Nucleic Acids Res. 42, D581-D591 (2014).

18. Turnbaugh, P. J. et al. The human microbiome project. Nature 449, 804-810 (2007).

19. D'Souza, G. \& Kost, C. Experimental evolution of metabolic dependency in bacteria. PLoS Genet. 12, e1006364 (2016).

20. Kim, S. C. et al. Variable phenotypes of enterocolitis in interleukin 10deficient mice monoassociated with two different commensal bacteria. Gastroenterology 128, 891-906 (2005).

21. Eun, C. S. et al. Induction of bacterial antigen-specific colitis by a simplified human microbiota consortium in gnotobiotic interleukin-10 $10^{-1-}$ mice. Infect. Immun. 82, 2239-2246 (2014).

22. Liu, B. et al. Combined in vivo/in vitro identification of human resident bacterial strains that selectively induce IL-10 and promote mucosal homeostasis. Gastroenterology https://doi.org/10.1016/s0016-5085(18)30763-7 (2018).
23. Liu, B. et al. Protective and potentially aggressive bacterial species occur simultaneously in healthy hosts. Gastroenterology https://doi.org/10.1016/ s0016-5085(19)36982-3 (2019).

24. Oka, A. et al. Tu1844-IL-10-independent protective activities of humanderived Clostridium strains in experimental colitis. Gastroenterology https:// doi.org/10.1016/s0016-5085(18)33470-x (2018).

25. Bibiloni, R., Simon, M. A., Albright, C., Sartor, B. \& Tannock, G. W. Analysis of the large bowel microbiota of colitic mice using PCR/DGGE. Lett. Appl. Microbiol. 41, 45-51 (2005).

26. Lupp, C. et al. Host-mediated inflammation disrupts the intestinal microbiota and promotes the overgrowth of Enterobacteriaceae. Cell Host Microbe 2, 119-129 (2007).

27. Mishima, Y. et al. Microbiota maintain colonic homeostasis by activating TLR2/MyD88/PI3K signaling in IL-10-producing regulatory B cells. J. Clin. Invest. https://doi.org/10.1172/JCI93820 (2019).

28. Neavin, D. R., Liu, D., Ray, B. \& Weinshilboum, R. M. The role of the aryl hydrocarbon receptor (AHR) in immune and inflammatory diseases. Int. J. Mol. Sci. https://doi.org/10.3390/ijms19123851 (2018).

29. Ramasundara, M., Leach, S. T., Lemberg, D. A. \& Day, A. S. Defensins and inflammation: the role of defensins in inflammatory bowel disease. $J$ Gastroenterol. Hepatol. 24, 202-208 (2009).

30. Magnusson, M. K. et al. Macrophage and dendritic cell subsets in IBD: ALDH + cells are reduced in colon tissue of patients with ulcerative colitis regardless of inflammation. Mucosal Immunol. 9, 171-182 (2016).

31. Darfeuille-Michaud, A. et al. High prevalence of adherent-invasive Escherichia coli associated with ileal mucosa in Crohn's disease. Gastroenterology 127, 412-421 (2004).

32. Gevers, D. et al. The treatment-naive microbiome in new-onset Crohn's disease. Cell Host Microbe 15, 382-392 (2014).

33. Boudeau, J., Glasser, A. L., Masseret, E., Joly, B. \& Darfeuille-Michaud, A. Invasive ability of an Escherichia coli strain isolated from the ileal mucosa of a patient with Crohn's disease. Infect. Immunity 67, 4499-4509 (1999).

34. Rogala, A. R., Oka, A. \& Sartor, R. B. Strategies to dissect host-microbial immune interactions that determine mucosal homeostasis vs. intestinal inflammation in Gnotobiotic mice. Front. Immunol. 11, 214 (2020).

35. Arpaia, N. et al. Metabolites produced by commensal bacteria promote peripheral regulatory T-cell generation. Nature 504, 451-455 (2013).

36. Smith, P. M. et al. The microbial metabolites, short-chain fatty acids, regulate colonic Treg cell homeostasis. Science 341, 569-573 (2013).

37. Bansal, T. et al. Differential effects of epinephrine, norepinephrine, and indole on Escherichia coli O157:H7 chemotaxis, colonization, and gene expression. Infect. Immun. 75, 4597-4607 (2007).

38. Bansal, T., Alaniz, R. C., Wood, T. K. \& Jayaraman, A. The bacterial signal indole increases epithelial-cell tight-junction resistance and attenuates indicators of inflammation. Proc. Natl Acad. Sci. USA 107, 228-233 (2010).

39. Duboc, H. et al. Connecting dysbiosis, bile-acid dysmetabolism and gut inflammation in inflammatory bowel diseases. Gut 62, 531-539 (2013).

40. Kortman, G. A., Raffatellu, M., Swinkels, D. W. \& Tjalsma, H. Nutritional iron turned inside out: intestinal stress from a gut microbial perspective. FEMS Microbiol. Rev. 38, 1202-1234 (2014).

41. Field, D., Cotter, P. D., Hill, C. \& Ross, R. P. Bioengineering lantibiotics for therapeutic success. Front. Microbiol. 6, 1363 (2015).

42. Oka, A. et al. A healthy human fecal specimen includes multiple E. Coli, Klebsiella Pneumoniae and Enterobacter strains with variable abilities to activate Th1 cells and induce colitis. Gastroenterology 156, S-246-S-247 (2019).

43. Vich Vila, A. et al. Gut microbiota composition and functional changes in inflammatory bowel disease and irritable bowel syndrome. Sci. Transl. Med. https://doi.org/10.1126/scitranslmed.aap8914 (2018).

44. Gilbert J. v. d. L. D. \& Taghavi S. Immune response modulation using live biotherapeutics, for conditions such as allergy desensitization. USA patent (2016).

45. Inoue, D., Tsujimoto, G. \& Kimura, I. Regulation of energy homeostasis by GPR41. Front. Endocrinol. (Lausanne) 5, 81 (2014).

46. Khaloian, S. et al. Mitochondrial impairment drives intestinal stem cell transition into dysfunctional Paneth cells predicting Crohn's disease recurrence. Gut https://doi.org/10.1136/gutjnl-2019-319514 (2020).

47. Song, $X$. et al. Microbial bile acid metabolites modulate gut RORgamma $(+)$ regulatory T cell homeostasis. Nature 577, 410-415 (2020).

48. Hang, S. et al. Bile acid metabolites control TH17 and Treg cell differentiation. Nature 576, 143-148 (2019).

49. Pandiyan, P. et al. The role of IL-15 in activating STAT5 and fine-tuning IL-17A production in CD4 T lymphocytes. J. Immunol. 189, 4237-4246 (2012).

50. Hubbard, T. D., Murray, I. A. \& Perdew, G. H. Indole and tryptophan metabolism: endogenous and dietary routes to Ah receptor activation. Drug Metab. Dispos. 43, 1522-1535 (2015). 
51. Venkatesh, M. et al. Symbiotic bacterial metabolites regulate gastrointestinal barrier function via the xenobiotic sensor PXR and Toll-like receptor 4 Immunity 41, 296-310 (2014).

52. Gutierrez-Vazquez, C. \& Quintana, F. J. Regulation of the immune response by the aryl hydrocarbon receptor. Immunity 48, 19-33 (2018).

53. Zhou, X. et al. PPARalpha-UGT axis activation represses intestinal FXRFGF15 feedback signalling and exacerbates experimental colitis. Nat. Commun. 5, 4573 (2014).

54. Tai, Y. T. et al. APRIL signaling via TACI mediates immunosuppression by T regulatory cells in multiple myeloma: therapeutic implications. Leukemia 33, 426-438 (2019).

55. Xie, M. M. et al. Bcl6 promotes follicular helper T-cell differentiation and PD1 expression in a Blimp1-independent manner in mice. Eur. J. Immunol. 47, 1136-1141 (2017).

56. Tan, Z. et al. Differentially expressed genes in the caecal and colonic mucosa of Landrace finishing pigs with high and low food conversion ratios. Sci. Rep. 7, 14886 (2017).

57. Hashimoto, T. et al. ACE2 links amino acid malnutrition to microbial ecology and intestinal inflammation. Nature 487, 477-481 (2012).

58. Wang, J. et al. ACE2 expression by colonic epithelial cells is associated with viral infection, immunity and energy metabolism. medRxiv preprint https:// doi.org/10.1101/2020.02.05.20020545 (2020).

59. Serino, M., Luche, E., Chabo, C., Amar, J. \& Burcelin, R. Intestinal microflora and metabolic diseases. Diabetes Metab. 35, 262-272 (2009).

60. van der Lelie, D. \& Taghavi, S. COVID-19 and the gut microbiome: more than a gut feeling. mSystems https://doi.org/10.1128/mSystems.00453-20 (2020).

61. Dess, N. K., Chapman, C. D., Fouladi, F., Fodor, A. A. \& Lyte, M. "Us vs. Them" pair housing: effects on body weight, open field behavior, and gut microbiota in rats selectively bred on a taste phenotype. Physiol. Behav. 223, 112975 (2020).

62. Nurk, S. et al. Assembling single-cell genomes and mini-metagenomes from chimeric MDA products. J. Comput. Biol. 20, 714-737 (2013).

63. Seemann, T. Prokka: rapid prokaryotic genome annotation. Bioinformatics $\mathbf{3 0}$ 2068-2069 (2014).

64. Aziz, R. K. et al. The RAST Server: rapid annotations using subsystems technology. BMC Genomics 9, 75 (2008).

65. Overbeek, R. et al. The SEED and the rapid annotation of microbial genomes using subsystems technology (RAST). Nucleic Acids Res. 42, D206-D214 (2014).

66. Brettin, T. et al. RASTtk: a modular and extensible implementation of the RAST algorithm for building custom annotation pipelines and annotating batches of genomes. Sci. Rep. 5, 8365 (2015).

67. Bolger, A. M., Lohse, M. \& Usadel, B. Trimmomatic: a flexible trimmer for Illumina sequence data. Bioinformatics 30, 2114-2120 (2014).

68. Menzel, P., Ng, K. L. \& Krogh, A. Fast and sensitive taxonomic classification for metagenomics with Kaiju. Nat. Commun. 7, 11257 (2016).

69. Blin, K. et al. antiSMASH 5.0: updates to the secondary metabolite genome mining pipeline. Nucleic Acids Res. 47, W81-W87 (2019).

70. Henry, C. S. et al. High-throughput generation, optimization and analysis of genome-scale metabolic models. Nat. Biotechnol. 28, 977-982 (2010).

71. Pritchard, L., Glover, R. H., Humpris, S., Elphinstone J. G. \& Toth, I. K. Genomics and taxonomy in diagnostics for food security: soft-rotting enterobacterial plant pathogens. Anal. Methods https://doi.org/10.1039/ c5ay02550h (2016).

72. Zheng, X. et al. A targeted metabolomic protocol for short-chain fatty acids and branched-chain amino acids. Metabolomics 9, 818-827 (2013).

73. Alnouti, Y., Csanaky, I. L. \& Klaassen, C. D. Quantitative-profiling of bile acids and their conjugates in mouse liver, bile, plasma, and urine using LCMS/MS. J. Chromatogr. B Anal. Technol. Biomed. Life Sci. 873, 209-217 (2008).
74. Swann, J. R. et al. Systemic gut microbial modulation of bile acid metabolism in host tissue compartments. Proc. Natl Acad. Sci. USA 108(Suppl 1), 4523-4530 (2011).

\section{Acknowledgements}

We would like to thank Jeremy Herzog and Lisa Holt for technical assistance. The work was funded by Gusto Global LLC. Germ-free mice were provided by NIH grants P40OD010995; P30DK034987; P01DK094779, and the Crohn's and Colitis Foundation. Instrumentation and resources for metabolomics work was partially supported by NIH grants R01ES024950; P30ES010126; R03ES032067, and P42ES031007.

\section{Author contributions}

D.v.d.L. conceived the study and with RBS designed experiments and wrote the manuscript; A.O. and J.U. executed the GUT-103 and GUT-108 animal studies and tissue analyses and helped prepare figures and methods; S.T. oversaw the strain isolation and characterization; T.J.F., K.E.M., and S.D.W. performed strain isolation, characterization, and genome sequencing; B.L. provided assistance to the animal studies; M.A. performed cytokine PCRs; Y.L., L.C., and K.L. performed the metabolite analysis; L.O. and C.H. performed the bioinformatics in support of GUT-103 and GUT-108 design. All authors provided significant input to the manuscript.

\section{Competing interests}

D.v.d.L., S.T., and C.H. are co-founders and shareholders with a personal financial interest in Gusto Global LLC. The results described in this manuscript support PCT patent application WO 2018/187272 Al and provisional patent application US63,094,655. R.B.S. received research grant support from Gusto Global LLC and serves as an unpaid consultant to the company. The other authors do not have competing interests.

\section{Additional information}

Supplementary information The online version contains supplementary material available at https://doi.org/10.1038/s41467-021-23460-x.

Correspondence and requests for materials should be addressed to D.v.d.L.

Peer review information:Nature Communications thanks the anonymous reviewers for their contribution to the peer review of this work.

Reprints and permission information is available at http://www.nature.com/reprints

Publisher's note Springer Nature remains neutral with regard to jurisdictional claims in published maps and institutional affiliations.

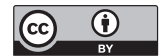

Open Access This article is licensed under a Creative Commons Attribution 4.0 International License, which permits use, sharing, adaptation, distribution and reproduction in any medium or format, as long as you give appropriate credit to the original author(s) and the source, provide a link to the Creative Commons license, and indicate if changes were made. The images or other third party material in this article are included in the article's Creative Commons license, unless indicated otherwise in a credit line to the material. If material is not included in the article's Creative Commons license and your intended use is not permitted by statutory regulation or exceeds the permitted use, you will need to obtain permission directly from the copyright holder. To view a copy of this license, visit http://creativecommons.org/ licenses/by/4.0/

(C) The Author(s) 2021 